\title{
ANALYSIS OF A CLASS OF NONCONFORMING FINITE ELEMENTS FOR CRYSTALLINE MICROSTRUCTURES
}

\author{
PETR KLOUČEK, BO LI AND MITCHELL LUSKIN
}

\begin{abstract}
An analysis is given for a class of nonconforming Lagrange-type finite elements which have been successfully utilized to approximate the solution of a variational problem modeling the deformation of martensitic crystals with microstructure. These elements were first proposed and analyzed in 1992 by Rannacher and Turek for the Stokes equation. Our analysis highlights the features of these elements which make them effective for the computation of microstructure. New results for superconvergence and numerical quadrature are also given.
\end{abstract}

\section{INTRODUCTION}

Recent years have seen the development of a continuum theory for martensitic crystals based on the minimization of the Ericksen-James elastic energy $[2,3,13,14$, $17,19]$. The elastic energy density attains a minimum value at several symmetryrelated deformation gradients. Thus, the deformations of energy-minimizing sequences often exhibit a microstructure - the simplest of which are fine-scale layers in which the deformation gradient is nearly constant and across which the deformation gradient oscillates between the energy wells - to allow the effective energy of a deformation to be that of a macroscopic or relaxed energy. Further, the parallel planes defining the layering in the microstructure are constrained by the symmetry of the energy density to be a member of a finite family of parallel planes.

If the deformation is constrained on the boundary, then the deformation cannot generally attain a minimum energy by forming a microstructure with layers of nonzero thickness [3]. Rather, minimizing sequences of deformations must be constructed from layers with a thickness which converges to zero. Such minimizing sequences define the solution to the variational problems. They can be described physically by the notion of microstructure and mathematically by the Young measure $[2,3,17,19]$.

When an energy minimizing deformation is sought in a finite element space, the fineness of the layers is limited not only by the mesh size, but also by the nature of the finite element used. The most accurate finite element spaces will be those which

Received by the editor March 8, 1994 and, in revised form, May 30, 1995.

1991 Mathematics Subject Classification. Primary 65N15, 65N30, 35J20, 35J70, 73V25.

Key words and phrases. Nonconforming finite element, error estimate, superconvergence, numerical quadrature.

This work was supported in part by the NSF through grant DMS 911-1572, by the AFOSR through grant AFOSR-91-0301, by the ARO through grants DAAL03-89-G-0081 and DAAL0392-G-0003, and by a grant from the Minnesota Supercomputer Institute. 
can approximate microstructures with the most fine-scale layers possible on meshes which are oriented arbitrarily with respect to the layers defining the microstructure.

Several approaches have been developed for the finite element approximation of microstructure. The most commonly used finite element spaces are the conforming spaces with continuous deformations which are either piecewise linear or multilinear with respect to some mesh. Although these spaces can approximate well microstructure with layers which are oriented with respect to the mesh, we have had difficulty approximating microstructure with these conforming spaces when the layers are not oriented with respect to the mesh. We have not generally been able to obtain solutions with conforming spaces which have a layer thickness of less than three elements if the grid is not oriented so that the planes across which the gradients of the deformations in the conforming finite element space are allowed to be discontinuous are not parallel to the layers.

Two alternative methods have been developed to allow microstructure to be approximated on meshes which are not aligned with the microstructure. The first method was that of reduced integration of the multilinear element $[8,9,12]$. This method has been effectively used to compute microstructure with fine-scale layers on meshes which are not oriented with respect to the microstructure. For Laplace's equation on a uniform grid, the deformation computed with this method can be shown to converge strongly, but the deformation gradients do not converge strongly. This would not be an effective method for the minimization of a quasi-convex energy, however this method can be used effectively with the nonconvex Ericksen-James energy since its energy-minimizing deformations converge strongly while its gradients do not converge strongly. Most importantly, numerical experiments indicate the convergence of the microstructure or Young measure for the piecewise constant projection of the gradients of the deformation.

The approach analyzed here is that given by a family of nonconforming finite elements. The use of nonconforming finite elements is intuitively appealing for problems with microstructure because the admissible deformations have more flexibility to approximate oscillatory functions. The nonconforming elements that we study in this paper were first proposed and analyzed by Rannacher and Turek for solving the Stokes problem [24]. Recently, we have used these finite elements to simulate the deformation of martensitic crystals with microstructure [18], and we found that with a suitable numerical quadrature they produce a very robust approximation method. Our analysis demonstrates that the deformation, as well as the deformation gradient, converges strongly for second-order, linear elliptic problems.

The first version of the considered elements is a finite element defined on rectangles (respectively, rectangular parallelepipeds) with degrees of freedom given by the values at midpoints of edges of the rectangles (respectively, the centers of the faces of the rectangular parallelepipeds). The second version is a finite element defined on rectangles (respectively, rectangular parallelepipeds) with the degrees of freedom given by the averages over the four edges of the rectangles (respectively, the six faces of the rectangular parallelepipeds).

Unlike most other nonconforming finite elements, these elements do not have any conforming counterparts. Consequently, the error analysis is nontrivial. We prove error estimates for these finite element approximations in both the $H^{1}$ and the $L^{2}$ norms. Our analysis contributes to the understanding of these elements by emphasizing their relation to the conforming multilinear elements. We also give new 
superconvergence estimates for the error of the deformation gradient. In view of practical computations, especially for the computation of material microstructures, we also give an analysis of the effect of the numerical integration.

The convergence of the approximation of the microstructure of the deformation gradient of a crystal by continuous, piecewise linear finite element methods has been proven in $[10,11]$ for one-dimensional model problems for norms which measure the weak convergence of nonlinear functions of the deformation gradient, and the convergence of the three-dimensional approximation of the microstructure of the magnetization in the micromagnetics model for ferromagnetics has been proven for related norms [22]. These norms are stronger than the $L^{2}$ norm, which does not control oscillations in the gradient, and are weaker than the $H^{1}$ norm, which does not allow oscillations in the gradient for convergent sequences. The above analyses and the multidimensional analysis in $[5,6]$ proceed by demonstrating that the deformation gradient (or magnetization in the micromagnetics problem) converges weakly and that the approximate deformation gradient (or magnetization) must lie in arbitrarily small neighborhoods of the minima of the energy density. Thus far, these techniques have not yet made possible the rigorous analysis of the numerical approximation of microstructure for realistic, multidimensional models for the deformation of crystals $[2,3,9]$.

Throughout this paper we will mostly focus on the three-dimensional approximations, although similar results hold in two dimensions. For simplicity, let $\Omega=\left(0, L_{1}\right) \times\left(0, L_{2}\right) \times\left(0, L_{3}\right)$ be a rectangular parallelepiped with faces parallel to the coordinate planes. The points of $\Omega$ will be denoted by $(x, y, z)$ or by $\left(x_{1}, x_{2}, x_{3}\right)$ as appropriate. Results similar to those presented in this paper are valid for domains which are the union of rectangular parallelepipeds except that the rate of convergence in the $L^{2}$ norm may be reduced since the regularity of the solution of the dual problem with $L^{2}$ data may be reduced.

We consider the following divergence-type second-order elliptic boundary value problem,

$$
\begin{aligned}
\mathcal{L} u \equiv-\frac{\partial}{\partial x}\left(a_{1} \frac{\partial u}{\partial x}\right)-\frac{\partial}{\partial y}\left(a_{2} \frac{\partial u}{\partial y}\right)-\frac{\partial}{\partial z}\left(a_{3} \frac{\partial u}{\partial z}\right)+c u & =f, & & \text { in } \Omega, \\
u & =0, & & \text { on } \partial \Omega,
\end{aligned}
$$

where $a_{1}, a_{2}, a_{3} \in W^{1, \infty}(\Omega), a_{1}, a_{2}, a_{3} \geq a_{0}=$ constant $>0$, a.e. $\Omega, c \in L^{\infty}(\Omega)$, $c \geq 0$, a.e. $\Omega, f \in L^{2}(\Omega)$. We define $a(\cdot, \cdot): H^{1}(\Omega) \times H^{1}(\Omega) \longrightarrow \mathbb{R}$ by

$$
a(v, w) \equiv \int_{\Omega}\left(a_{1} \frac{\partial v}{\partial x} \frac{\partial w}{\partial x}+a_{2} \frac{\partial v}{\partial y} \frac{\partial w}{\partial y}+a_{3} \frac{\partial v}{\partial z} \frac{\partial w}{\partial z}+c v w\right) d x d y d z .
$$

It is obvious that $a(\cdot, \cdot)$ is symmetric, continuous and bilinear. Furthermore, by the Poincaré inequality, $a(\cdot, \cdot)$ is $H_{0}^{1}(\Omega)$-elliptic. We denote by $(\cdot, \cdot)$ the $L^{2}(\Omega)$ inner product. The existence and uniqueness of the solution to the problem (1.1) follow from the Lax-Milgram lemma. The following theorem gives the regularity of the solution $[15,16]$.

Theorem 1.1. For any $f \in L^{2}(\Omega)$, there exists a unique $u \in H_{0}^{1}(\Omega) \cap H^{2}(\Omega)$ such that

$$
a(u, v)=(f, v), \quad \forall v \in H_{0}^{1}(\Omega) .
$$


Furthermore, there holds the a priori estimate

$$
\|u\|_{2, \Omega} \leq C\|f\|_{0, \Omega},
$$

where $C=C\left(a_{1}, a_{2}, a_{3}, c, \Omega\right)>0$ is a constant.

The rest of this paper is organized as follows. In $\S 2$ we define the finite elements and their corresponding finite element spaces based on a rectangular partition of $\Omega$. We then prove a Poincaré-type inequality for the test functions. In $\S 3$ we give the error estimates in both the $H^{1}$ and the $L^{2}$ norms. In $\S 4$, we discuss the relation of the considered finite elements to multilinear finite elements. In $\S 5$, we give a superconvergence estimate for the gradients based on cubic partitions. Finally, in $\S 6$, we apply numerical quadrature to the finite element approximations, and we study the rates of convergence for several resulting schemes.

\section{The Finite ELEMENTS}

The first finite element is defined by the triple $\left(Q, P_{Q}, \Sigma_{Q}^{p}\right)$, where $Q \equiv[a-r$, $a+r] \times[b-s, b+s] \times[c-t, c+t]$ is a rectangular parallelepiped with its center at $(a, b, c)$ and the lengths of its edges $2 r, 2 s, 2 t$, where $r, s, t>0$,

$$
\begin{aligned}
& P_{Q}=\operatorname{Span}\left\{1, x, y, z,\left(\frac{x}{r}\right)^{2}-\left(\frac{y}{s}\right)^{2},\left(\frac{x}{r}\right)^{2}-\left(\frac{z}{t}\right)^{2}\right\}, \\
& \Sigma_{Q}^{p}=\left\{q\left(M_{i}\right): i=1, \ldots, 6\right\},
\end{aligned}
$$

where $M_{i}, i=1, \ldots, 6$, are the centers of the faces of $Q$. This Lagrange-type element is well defined since it is easy to verify that $\Sigma_{Q}^{p}$ is $P_{Q}$-unisolvent, i.e., for any given $\alpha_{i} \in \mathbb{R}, i=1, \ldots, 6$, there exists a unique $q \in P_{Q}$ such that

$$
q\left(M_{i}\right)=\alpha_{i}, \quad i=1, \ldots, 6 .
$$

We define $\varphi_{i}=\varphi_{i}(x, y, z) \in P_{Q}, i=1, \ldots, 6$, such that

$$
\varphi_{i}\left(M_{j}\right)=\delta_{i j}, \quad i, j=1, \ldots, 6,
$$

by permuting the terms $(x-a) / r,(y-b) / s$ and $(z-c) / t$ in the polynomial

$$
\varphi(x, y, z)=-\frac{1}{6}\left(\frac{x-a}{r}\right)^{2}-\frac{1}{6}\left(\frac{y-b}{s}\right)^{2}+\frac{1}{3}\left(\frac{z-c}{t}\right)^{2}+\epsilon \frac{z-c}{2 t}+\frac{1}{6},
$$

where $\epsilon= \pm 1$. Thus, it follows that $\left\{\varphi_{i}\right\}_{i=1}^{6}$ is the standard basis for the finite element $\left(Q, P_{Q}, \Sigma_{Q}^{p}\right)$. We then define the affine family of finite elements $\left(R, P_{R}, \Sigma_{R}^{p}\right)$, where $R$ is a rectangular parallelepiped. We note that in general $\nabla \cdot \nabla \phi \neq 0$ for $\phi \in P_{Q}$ unless $r=s=t$.

Next, we define the averaged version of the preceding finite element to be the triple $\left(Q, P_{Q}, \Sigma_{Q}^{a}\right)$. The polynomial space $P_{Q}$ is the same as defined in (2.1) and the set of degrees of freedom is defined by

$$
\Sigma_{Q}^{a}=\left\{f_{F_{i}} q d S: i=1, \ldots, 6\right\}
$$


where $F_{i}, i=1, \ldots, 6$, are the faces of the rectangular parallelepiped $Q$ and

$$
f_{F} \equiv \frac{1}{|F|} \int_{F}
$$

for faces $F \subset \partial Q$, where $|F|$ denotes the area of the face $F$. This finite element is well defined since $\Sigma_{Q}^{a}$ is $P_{Q}$-unisolvent. This can be easily checked by considering the six polynomials $\psi_{i}=\psi_{i}(x, y, z), i=1, \ldots, 6$, obtained by permuting the terms $(x-a) / r,(y-b) / s$ and $(z-c) / t$ in the polynomial

$$
\psi(x, y, z)=-\frac{1}{4}\left(\frac{x-a}{r}\right)^{2}-\frac{1}{4}\left(\frac{y-b}{s}\right)^{2}+\frac{1}{2}\left(\frac{z-c}{t}\right)^{2}+\epsilon \frac{z-c}{2 t}+\frac{1}{6},
$$

where $\epsilon= \pm 1$. It is obvious that $\psi_{i} \in P_{Q}, i=1, \ldots, 6$, and it is easily checked that with a suitable labeling of the indices,

$$
f_{F_{i}} \psi_{j} d S=\delta_{i j}
$$

Thus, $\left\{\psi_{i}\right\}_{i=1}^{6}$ is the standard basis for the finite element $\left(Q, P_{Q}, \Sigma_{Q}^{a}\right)$. Again, we define the affine family of finite elements $\left(R, P_{R}, \Sigma_{R}^{a}\right)$, where $R$ is a rectangular parallelepiped.

To construct a rectangular partition $\tau_{h}$ of $\Omega$, we define one-dimensional partitions of $\left[0, L_{k}\right]$, for $k=1,2,3$, by

$$
0=x_{k}^{0}<x_{k}^{1}<\cdots<x_{k}^{m_{k}}=L_{k},
$$

where $m_{k}$ are positive integers. We then define the rectangular parallelepipeds

$R_{i_{1}, i_{2}, i_{3}} \equiv\left[x_{1}^{i_{1}-1}, x_{1}^{i_{1}}\right] \times\left[x_{2}^{i_{2}-1}, x_{2}^{i_{2}}\right] \times\left[x_{3}^{i_{3}-1}, x_{3}^{i_{3}}\right], \quad 1 \leq i_{1} \leq m_{1}, \ldots, 1 \leq i_{3} \leq m_{3}$,

and the rectangular partition

$$
\tau_{h} \equiv\left\{R_{i_{1}, i_{2}, i_{3}}: 1 \leq i_{1} \leq m_{1}, \ldots, 1 \leq i_{3} \leq m_{3}\right\}
$$

with the mesh size parameter $h$ defined by $h=\max \left\{h_{k}: 1 \leq k \leq 3\right\}$, where $h_{k} \equiv \max \left\{x_{k}^{i}-x_{k}^{i-1}: 1 \leq i \leq m_{k}\right\}$ is the maximal discretization size in the $k$ th coordinate direction for $k=1,2,3$.

For the first finite element, we define the set of nodal points $N_{h}$ to be the set of all the centers of faces of elements in $\tau_{h}$. The finite element spaces over the partition $\tau_{h}$ are then defined respectively to be

$$
\begin{aligned}
& V_{h}^{p} \equiv\left\{v_{h} \in L^{2}(\Omega):\left.v_{h}\right|_{R} \in P_{R}, \forall R \in \tau_{h} ; \text { adjoining } v_{h}\right. \text { have the same } \\
& \left.\quad \text { values at shared nodal points, i.e., } v_{h} \text { is continuous on } N_{h}\right\}, \\
& V_{h}^{a} \equiv\left\{v_{h} \in L^{2}(\Omega):\left.v_{h}\right|_{R} \in P_{R}, \forall R \in \tau_{h} ;\right. \\
& \left.\left.\quad \int_{F} v_{h}\right|_{R^{\prime}} d S=\left.\int_{F} v_{h}\right|_{R^{\prime \prime}} d S, \forall \text { faces } F=\partial R^{\prime} \cap \partial R^{\prime \prime} \neq \emptyset, R^{\prime}, R^{\prime \prime} \in \tau_{h}\right\} .
\end{aligned}
$$


To solve the Dirichlet problem (1.1), we define

$$
\begin{aligned}
V_{0 h}^{p} & \equiv\left\{v_{h} \in V_{h}^{p}: v_{h}=0 \text { on } N_{h} \cap \partial \Omega\right\}, \\
V_{0 h}^{a} & \equiv\left\{v_{h} \in V_{h}^{a}: \int_{F} v_{h} d S=0, \forall \text { faces } F \subset \partial R \cap \partial \Omega \neq \emptyset, R \in \tau_{h}\right\} .
\end{aligned}
$$

It is obvious that all of the spaces $V_{h}^{p}, V_{0 h}^{p}, V_{h}^{a}$ and $V_{0 h}^{a}$ are finite-dimensional subspaces of $L^{2}(\Omega)$. They are also affine finite element spaces [7]. For $v_{h} \in V_{h}^{p}$ (or $\left.V_{0 h}^{p}, V_{h}^{a}, V_{0 h}^{a}\right)$, we have in general that $v_{h} \notin C(\bar{\Omega})$ since $v_{h}$ is continuous necessarily only at centers (or at some other points in the case of the $V_{h}^{a}$-approximation) of faces of adjacent elements. Therefore, $V_{h}^{p}$ (or $\left.V_{0 h}^{p}, V_{h}^{a}, V_{0 h}^{a}\right) \nsubseteq \subset C(\bar{\Omega})$, and, hence, $V_{h}^{p}$ (or $\left.V_{0 h}^{p}, V_{h}^{a}, V_{0 h}^{a}\right) \nsubseteq H^{1}(\Omega)$. Thus, in view of solving a second-order elliptic boundary value problem, the finite elements are nonconforming.

For convenience, we define for an integer $k \geq 0$ and $p \in[1, \infty]$ the space

$$
W_{h}^{k, p}(\Omega) \equiv\left\{v \in L^{p}(\Omega):\left.v\right|_{R} \in W^{k, p}(R), \forall R \in \tau_{h}\right\},
$$

and equip $W_{h}^{k, p}(\Omega)$ with the following seminorm and norm:

$$
\begin{aligned}
|\cdot|_{k, p, h} & \equiv \begin{cases}\left(\sum_{R \in \tau_{h}}|\cdot|_{k, p, R}^{p}\right)^{\frac{1}{p}}, & \text { if } 1 \leq p<\infty, \\
\max _{R \in \tau_{h}}|\cdot|_{k, \infty, R}, & \text { if } p=\infty ;\end{cases} \\
\|\cdot\|_{k, p, h} & \equiv \begin{cases}\left(\sum_{R \in \tau_{h}}\|\cdot\|_{k, p, R}^{p}\right)^{\frac{1}{p}}, & \text { if } 1 \leq p<\infty, \\
\max _{R \in \tau_{h}}\|\cdot\|_{k, \infty, R}, & \text { if } p=\infty,\end{cases}
\end{aligned}
$$

where, for $R \in \tau_{h},|\cdot|_{k, p, R}$ and $\|\cdot\|_{k, p, R}$ are the usual seminorm and norm on the Sobolev space $W^{k, p}(R)$ [1]. If $p=2$ we write $H_{h}^{k}(\Omega)$ for $W_{h}^{k, p}(\Omega)$ and omit $p$ in all the above seminorm and norm expressions.

Now it is obvious that $|\cdot|_{1, h}$ defines a norm on $V_{0 h}^{p}$ and $V_{0 h}^{a}$. We next prove a Poincaré-type inequality for functions in the finite element spaces $V_{0 h}^{p}$ and $V_{0 h}^{a}$. This inequality leads to the uniform $V_{0 h^{-}}^{p}$ and $V_{0 h^{-}}^{a}$-ellipticity, which is required in deriving the second Strang lemma [7, 25].

Theorem 2.1. For any $v_{h} \in V_{0 h}^{p} \cup V_{0 h}^{a}$, we have

$$
\left\|v_{h}\right\|_{0, \Omega} \leq \sqrt{6} h\left|v_{h}\right|_{1, h}+\sqrt{2} L_{k}\left\|\frac{\partial v_{h}}{\partial x_{k}}\right\|_{0, h}, \quad k=1,2,3 .
$$

Proof. Let us fix $v \equiv v_{h} \in V_{0 h}^{p} \cup V_{0 h}^{a}$. For any $\overline{\boldsymbol{x}}=\left(\bar{x}_{1}, \overline{\boldsymbol{x}}^{\prime}\right) \in \Omega$, where $\overline{\boldsymbol{x}}^{\prime}=\left(\bar{x}_{2}, \bar{x}_{3}\right)$, let $R=\left[x_{1}^{i_{1}-1}, x_{1}^{i_{1}}\right] \times R^{\prime}$, where $R^{\prime}=\left[x_{2}^{i_{2}-1}, x_{2}^{i_{2}}\right] \times\left[x_{3}^{i_{3}-1}, x_{3}^{i_{3}}\right]$, be such that $\overline{\boldsymbol{x}} \in R$. Without loss of generality, we assume $i_{1} \geq 2$. Denote by $v_{j}$ the restriction of $v$ on $\left[x_{1}^{j-1}, x_{1}^{j}\right] \times R^{\prime}$, for $j=1, \ldots, m_{1}$. If $v \in V_{0 h}^{a}$, we have

$$
\int_{R^{\prime}} v_{1}\left(x_{1}^{0}, \boldsymbol{x}^{\prime}\right) d \boldsymbol{x}^{\prime}+\sum_{j=1}^{i_{1}-1}\left[\int_{R^{\prime}} v_{j+1}\left(x_{1}^{j}, \boldsymbol{x}^{\prime}\right) d \boldsymbol{x}^{\prime}-\int_{R^{\prime}} v_{j}\left(x_{1}^{j}, \boldsymbol{x}^{\prime}\right) d \boldsymbol{x}^{\prime}\right]=0 .
$$


Consequently, there exists $\boldsymbol{z}^{\prime}=\left(z_{2}^{\prime}, z_{3}^{\prime}\right) \in R^{\prime}$ so that

$$
v_{1}\left(x_{1}^{0}, \boldsymbol{z}^{\prime}\right)+\sum_{j=1}^{i_{1}-1}\left[v_{j+1}\left(x_{1}^{j}, \boldsymbol{z}^{\prime}\right)-v_{j}\left(x_{1}^{j}, \boldsymbol{z}^{\prime}\right)\right]=0 .
$$

Observe that on each element in $\tau_{h}$,

$$
\frac{\partial v}{\partial x_{k}} \in \operatorname{Span}\left\{1, x_{k}\right\}, \quad k=1,2,3 .
$$

By (2.9) and (2.10), we have

$$
\begin{aligned}
v(\overline{\boldsymbol{x}}) & =v_{i_{1}}\left(\bar{x}_{1}, \overline{\boldsymbol{x}}^{\prime}\right)-v_{i_{1}}\left(x_{1}^{i_{1}-1}, \boldsymbol{z}^{\prime}\right)+\sum_{j=1}^{i_{1}-1}\left[v_{j}\left(x_{1}^{j}, \boldsymbol{z}^{\prime}\right)-v_{j}\left(x_{1}^{j-1}, \boldsymbol{z}^{\prime}\right)\right] \\
& =\sum_{k=1}^{3} \int_{z_{k}^{\prime}}^{\bar{x}_{k}} \frac{\partial v_{i_{1}}(\boldsymbol{x})}{\partial x_{k}} d x_{k}+\sum_{j=1}^{i_{1}-1} \int_{x_{1}^{j-1}}^{x_{1}^{j}} \frac{\partial v_{j}(\boldsymbol{x})}{\partial x_{1}} d x_{1},
\end{aligned}
$$

where $z_{1}^{\prime}=x_{1}^{i_{1}-1}$. This is also true for $v \in V_{0 h}^{p}$ if we choose $\boldsymbol{z}^{\prime} \in R^{\prime}$ so that $\left(x_{1}^{0}, z^{\prime}\right)$ is the center of the face $\left\{x_{1}^{0}\right\} \times R^{\prime}$ of the element $\left[x_{1}^{0}, x_{1}^{1}\right] \times R^{\prime}$. It then follows from (2.11), (2.10) and the Cauchy-Schwarz inequality that

$$
|v(\overline{\boldsymbol{x}})|^{2} \leq 6 h \sum_{k=1}^{3} \int_{x_{k}^{i_{k}-1}}^{x_{k}^{i_{k}}}\left|\frac{\partial v_{i_{1}}(\boldsymbol{x})}{\partial x_{k}}\right|^{2} d x_{k}+2 L_{1} \sum_{j=1}^{m_{1}} \int_{x_{1}^{j-1}}^{x_{1}^{j}}\left|\frac{\partial v_{j}(\boldsymbol{x})}{\partial x_{1}}\right|^{2} d x_{1} .
$$

Integrating (2.12) over $R$ and summing up the integrals over $R \in \tau_{h}$, we thus obtain (2.8) for $k=1$. The same argument applies to $k=2,3$.

\section{3. $H_{h}^{1}$ AND $L^{2}$ ERROR ESTIMATES}

We define $a_{h}(\cdot, \cdot): H_{h}^{1}(\Omega) \times H_{h}^{1}(\Omega) \longrightarrow \mathbb{R}$ by

$$
a_{h}(v, w) \equiv \sum_{R \in \tau_{h}} \int_{R}\left(a_{1} \frac{\partial v}{\partial x} \frac{\partial w}{\partial x}+a_{2} \frac{\partial v}{\partial y} \frac{\partial w}{\partial y}+a_{3} \frac{\partial v}{\partial z} \frac{\partial w}{\partial z}+c v w\right) d x d y d z .
$$

We also denote $V_{h}=V_{h}^{p}$ or $V_{h}^{a}$ and $V_{0 h}=V_{0 h}^{p}$ or $V_{0 h}^{a}$, respectively. It is clear that $a_{h}(\cdot, \cdot)$ is symmetric, continuous and bilinear. By Theorem 2.1, it is also uniform $V_{0 h}$-elliptic, i.e., there exists a constant $\alpha>0$, independent of $h$, such that

$$
a_{h}\left(v_{h}, v_{h}\right) \geq \alpha\left\|v_{h}\right\|_{1, h}^{2}, \quad \forall v_{h} \in V_{0 h} .
$$

Therefore, by the Lax-Milgram lemma, there exists a unique finite element approximation $u_{h} \in V_{0 h}$ such that

$$
a_{h}\left(u_{h}, v_{h}\right)=\left(f, v_{h}\right), \quad \forall v_{h} \in V_{0 h} .
$$

In the sequel, the rectangular partitions $\tau_{h}$ are always assumed to be quasiuniform, i.e., there exists a constant $\sigma>0$, independent of $h$, such that

$$
\min \left\{x_{k}^{i}-x_{k}^{i-1}: i=1, \ldots, m_{k}, k=1,2,3\right\} \geq \sigma h .
$$


We denote the Lagrange interpolation operator $I_{h}: C(\bar{\Omega}) \longrightarrow V_{h}$ to be either $I_{h}^{p}: C(\bar{\Omega}) \longrightarrow V_{h}^{p}$ or $I_{h}^{a}: C(\bar{\Omega}) \longrightarrow V_{h}^{a}$, which are defined respectively for $I_{h}^{p} v \in V_{h}^{p}$ and $I_{h}^{a} v \in V_{h}^{a}$ by

$$
\begin{array}{rlrl}
I_{h}^{p} v(M) & =v(M), & \forall M \in N_{h}, \\
\int_{F} I_{h}^{a} v d S & =\int_{F} v d S, \quad \forall \text { faces } F \subset \partial R \text { where } R \in \tau_{h},
\end{array}
$$

for any $v \in C(\bar{\Omega})$. We also use the same notation $I_{h}, I_{h}^{p}$ and $I_{h}^{a}$ to denote the restrictions of these operators over an element of the partition $\tau_{h}$.

We use the symbol $C$ to denote a generic constant varying with the context. This constant is always assumed to be independent of all the trial and test functions, the solution $u$ to (1.2) and the mesh size parameter $h$ unless the dependence is otherwise stated.

Let us recall the following well-known results on the estimates for interpolation errors and the inverse estimates for later use [7].

Theorem 3.1. For $k=0,1,2$, we have

$$
\begin{aligned}
\left\|I_{h} v-v\right\|_{k, R} \leq C h^{2-k}|v|_{2, R}, & \forall R \in \tau_{h}, \forall v \in H^{2}(R), \\
\left\|I_{h} v-v\right\|_{k, h} \leq C h^{2-k}|v|_{2, h}, & \forall v \in H_{h}^{2}(\Omega) .
\end{aligned}
$$

Theorem 3.2. Let $k$ and $l$ be two integers such that $0 \leq k \leq l \leq 2$. Then for any $R \in \tau_{h}$ and any $v_{h} \in V_{h}$ we have

$$
\begin{aligned}
& \left|v_{h}\right|_{l, R} \leq C h^{k-l}\left|v_{h}\right|_{k, R}, \\
& \left|v_{h}\right|_{l, h} \leq C h^{k-l}\left|v_{h}\right|_{k, h}, \\
& \left|v_{h}\right|_{l, \infty, R} \leq C h^{k-l-\frac{3}{2}}\left|v_{h}\right|_{k, R}, \\
& \left|v_{h}\right|_{l, \infty, h} \leq C h^{k-l-\frac{3}{2}}\left|v_{h}\right|_{k, h} .
\end{aligned}
$$

Our main results in this section are the error estimates for the finite element approximations in the $H_{h}^{1}(\Omega)$ and the $L^{2}(\Omega)$ norms.

Theorem 3.3. Let $u \in H_{0}^{1}(\Omega) \cap H^{2}(\Omega)$ and $u_{h} \in V_{0 h}$ be the solutions to (1.2) and (3.2), respectively. We have

$$
\left\|u-u_{h}\right\|_{m, h} \leq C h^{2-m}\|u\|_{2, \Omega}, \quad m=0,1 .
$$

To prove the theorem, we need some auxiliary lemmas.

Lemma 3.4. Let $R \in \tau_{h}$ and $F \subset \partial R$ be a face of $R$ and let $P_{0} \in R$ be an arbitrary point. Then the following estimates hold:

$$
\begin{array}{ll}
\left|w\left(P_{0}\right)-f_{R} w d x d y d z\right| \leq C h|w|_{1, \infty, R}, & \forall w \in W^{1, \infty}(R), \\
\int_{F}\left|w-f_{F} w d S\right|^{p} d S \leq C h^{2-\frac{p}{2}}|w|_{1, R}^{p}, & \forall w \in H^{1}(R), p=1,2, \\
\int_{\partial R} w^{2} d S \leq \frac{C}{h}|w|_{0, R}^{2}+C h|w|_{1, R}^{2}, & \forall w \in H^{1}(R) .
\end{array}
$$


Proof. The inequality (3.4) follows easily from the estimate

$$
\begin{aligned}
\left|w\left(P_{0}\right)-f_{R} w d x d y d z\right| & =\left|f_{R}\left[w\left(P_{0}\right)-w(x, y, z)\right] d x d y d z\right| \\
& \leq f_{R}\left|w\left(P_{0}\right)-w(x, y, z)\right| d x d y d z \\
& \leq f_{R}\left|P_{0}-(x, y, z)\right||w|_{1, \infty, R} d x d y d z
\end{aligned}
$$

for $w \in W^{1, \infty}(R)$.

Next, let $R=[a-r, a+r] \times[b-s, b+s] \times[c-t, c+t]$ and assume without loss of generality that $F=\{a+r\} \times[b-s, b+s] \times[c-t, c+t]$. Denote $\hat{R}=$ $[-1,1] \times[-1,1] \times[-1,1]$ and $\hat{F}=\{1\} \times[-1,1] \times[-1,1]$, and define the affine mapping $K_{R}: \hat{R} \longrightarrow R$ by $K(\xi, \eta, \zeta)=(x, y, z)$, where

$$
x=r \xi+a, \quad y=s \eta+b, \quad z=t \zeta+c .
$$

For any function $w=w(P), P \in R$, set $\hat{w}=w \circ K_{R}$. Now by the quasi-uniformity of $\tau_{h}$ and the trace theorem [1] we get that

$$
\int_{F}\left|w-f_{F} w d S\right|^{p} d S=s t \int_{\hat{F}}\left|\hat{w}-f_{\hat{F}} \hat{w} d \hat{S}\right|^{p} d \hat{S} \leq C h^{2}\|\hat{w}\|_{1, \hat{R}}^{p} .
$$

Replacing $w$ by $w+c$ in (3.8) with $c$ any constant, we have by the Bramble-Hilbert lemma [7, Theorem 4.1.3] that

$$
\int_{F}\left|w-f_{F} w d S\right|^{p} d S \leq C h^{2} \inf _{\hat{c}=\text { constant }}\|\hat{w}+\hat{c}\|_{1, \hat{R}}^{p} \leq C h^{2}|\hat{w}|_{1, \hat{R}}^{p} \leq C h^{2-\frac{p}{2}}|w|_{1, R}^{p} \text {. }
$$

This proves (3.5).

Finally, by the transformation (3.7), the quasi-uniformity of $\tau_{h}$ and the trace theorem, we have

$$
\begin{aligned}
\int_{\partial R} w^{2} d S & \leq C h^{2} \int_{\partial \hat{R}} \hat{w}^{2} d \hat{S} \leq C h^{2}\left[\int_{\hat{R}} \hat{w}^{2} d \xi d \eta d \zeta+\int_{\hat{R}}|\nabla \hat{w}|^{2} d \xi d \eta d \zeta\right] \\
& \leq C h^{2}\left[h^{-3} \int_{R} w^{2} d x d y d z+h^{-1} \int_{R}|\nabla w|^{2} d x d y d z\right]
\end{aligned}
$$

leading to (3.6).

In what follows, for $R \in \tau_{h}$ and a face $F \subset \partial R$, we define the functional $T_{F}$ by either $T_{F}(w)=w\left(M_{F}\right)$ for $w \in C(F)$, where $M_{F}$ is the center of the face $F$, when considering the $V_{h}^{p}$-approximation, or by $T_{F}(w)=f_{F} w d S$ for $w \in L^{2}(F)$ when considering the $V_{h}^{a}$-approximation.

Lemma 3.5. For any $R \in \tau_{h}$ and any face $F \subset \partial R$, we have

$$
\int_{F}\left[v_{h}-T_{F}\left(v_{h}\right)\right]^{2} d S \leq C h\left|v_{h}\right|_{1, R}^{2}, \quad \forall v_{h} \in V_{h} .
$$


Proof. Without loss of generality, we assume that $F=\{a+r\} \times[b-s, b+s] \times$ $[c-t, c+t]$. Let $P_{F} \equiv(a+r, \bar{y}, \bar{z}) \in F$ be such that $T_{F}\left(v_{h}\right)=v_{h}\left(P_{F}\right)$. Now, by the Cauchy-Schwarz inequality, the fact (2.10) that $\frac{\partial v_{h}}{\partial y}$ (respectively, $\frac{\partial v_{h}}{\partial z}$ ) depends only on $y$ (respectively, $z$ ), and the quasi-uniformity of the partitions $\tau_{h}$, we have

$$
\begin{aligned}
\int_{F}\left[v_{h}-T_{F}\left(v_{h}\right)\right]^{2} d S=\int_{b-s}^{b+s} \int_{c-t}^{c+t}\left[v_{h}(a+r, y, z)-v_{h}(a+r, \bar{y}, \bar{z})\right]^{2} d y d z \\
=\int_{b-s}^{b+s} \int_{c-t}^{c+t}\left[\int_{\bar{y}}^{y} \frac{\partial v_{h}\left(a+r, y^{\prime}, z\right)}{\partial y^{\prime}} d y^{\prime}+\int_{\bar{z}}^{z} \frac{\partial v_{h}\left(a+r, \bar{y}, z^{\prime}\right)}{\partial z^{\prime}} d z^{\prime}\right]^{2} d y d z \\
\leq \int_{b-s}^{b+s} \int_{c-t}^{c+t}\left\{2\left[\int_{\bar{y}}^{y} \frac{\partial v_{h}\left(a+r, y^{\prime}, z\right)}{\partial y^{\prime}} d y^{\prime}\right]^{2}\right. \\
\left.\quad+2\left[\int_{\bar{z}}^{z} \frac{\partial v_{h}\left(a+r, \bar{y}, z^{\prime}\right)}{\partial z^{\prime}} d z^{\prime}\right]^{2}\right\} d y d z \\
\leq \int_{b-s}^{b+s} \int_{c-t}^{c+t}\left\{\left.2|y-\bar{y}|\left|\int_{\bar{y}}^{y}\right| \frac{\partial v_{h}\left(a+r, y^{\prime}, z\right)}{\partial y^{\prime}}\right|^{2} d y^{\prime} \mid\right. \\
\left.\quad+\left.2|z-\bar{z}|\left|\int_{\bar{z}}^{z}\right| \frac{\partial v_{h}\left(a+r, \bar{y}, z^{\prime}\right)}{\partial z^{\prime}}\right|^{2} d z^{\prime} \mid\right\} d y d z \\
\leq \int_{b-s}^{b+s} \int_{c-t}^{c+t}\left\{4 s \int_{b-s}^{b+s}\left|\frac{\partial v_{h}}{\partial y}\right|^{2} d y+4 t \int_{c-t}^{c+t}\left|\frac{\partial v_{h}}{\partial z}\right|^{2} d z\right\} d y d z \\
\leq 4 s t\left(4 s \int_{b-s}^{b+s}\left|\frac{\partial v_{h}}{\partial y}\right|^{2} d y+4 t \int_{c-t}^{c+t}\left|\frac{\partial v_{h}}{\partial z}\right|^{2} d z\right) \leq C h\left|v_{h}\right|_{1, R}^{2},
\end{aligned}
$$

completing the proof.

Lemma 3.6. Let $R \in \tau_{h}$ and let $F \subset \partial R$ be a face of $R$. Then, for any trilinear function $w=w(x, y, z)$ on $R$, we have

$$
\int_{F}\left(w-I_{h} w\right) d S=0 \quad \text { and } \quad T_{F}\left(w-I_{h} w\right)=0 .
$$

Proof. Without loss of generality, let $R \in \tau_{h}$ and $F \subset \partial R$ be the same as in the proof of Lemma 3.5. If $w=1, x, y$ or $z$, then $I_{h} w=w$. Hence, (3.9) holds trivially. Now if $w$ is not linear but multilinear with respect to the variables $x-a, y-b$, and $z-c$, then a simple calculation shows that $I_{h} w=0, T_{F}(w)=0$, and (3.9) is true as well. Our proof is complete since all the trilinear functions are linear combinations of those functions tested above.

Now we are in a position to prove our theorem.

Proof of the $H_{h}^{1}$ error estimate. By Theorem $2.1, a_{h}(\cdot, \cdot)$ is uniformly $V_{0 h}$-elliptic. Hence, by the second Strang lemma [7, 25], we have

$$
\left\|u-u_{h}\right\|_{1, h} \leq C\left[\inf _{v_{h} \in V_{0 h}}\left\|u-v_{h}\right\|_{1, h}+\sup _{0 \neq v_{h} \in V_{0 h}} \frac{\left|d_{h}\left(u, v_{h}\right)\right|}{\left\|v_{h}\right\|_{1, h}}\right],
$$


where $d_{h}: H^{2}(\Omega) \times H_{h}^{1}(\Omega) \longrightarrow \mathbb{R}$ is the nonconforming error functional defined by

$$
d_{h}(\varphi, v)=a_{h}(\varphi, v)-(\mathcal{L} \varphi, v), \quad \varphi \in H^{2}(\Omega), v \in H_{h}^{1}(\Omega),
$$

and where $\mathcal{L}$ is the differential operator defined in (1.1). Since $u=0$ on $\partial \Omega$, and $u \in H^{2}(\Omega) \hookrightarrow C(\bar{\Omega})$, we have that $I_{h} u \in V_{0 h}$. Thus, Theorem 3.1 leads to the estimate

$$
\inf _{v_{h} \in V_{0 h}}\left\|u-v_{h}\right\|_{1, h} \leq\left\|u-I_{h} u\right\|_{1, h} \leq C h\|u\|_{2, \Omega} .
$$

To estimate the error functional $d_{h}(\cdot, \cdot)$, we fix an arbitrary function $v=v_{h} \in V_{0 h}$. Since $u \in H_{0}^{1}(\Omega) \cap H^{2}(\Omega)$ is the solution to (1.2), by integration by parts, we get

$$
\begin{aligned}
d_{h}(u, v) & =\sum_{R \in \tau_{h}} \int_{R}\left(a_{1} \frac{\partial u}{\partial x} \frac{\partial v}{\partial x}+a_{2} \frac{\partial u}{\partial y} \frac{\partial v}{\partial y}+a_{3} \frac{\partial u}{\partial z} \frac{\partial v}{\partial z}+c u v-f v\right) d x d y d z \\
& =\sum_{R \in \tau_{h}} \int_{\partial R} a_{1} \frac{\partial u}{\partial x} v n_{1} d S+\sum_{R \in \tau_{h}} \int_{\partial R} a_{2} \frac{\partial u}{\partial y} v n_{2} d S+\sum_{R \in \tau_{h}} \int_{\partial R} a_{3} \frac{\partial u}{\partial z} v n_{3} d S \\
& \equiv I_{1}+I_{2}+I_{3},
\end{aligned}
$$

where $n=\left(n_{1}, n_{2}, n_{3}\right)$ is the unit outer normal to the boundary $\partial R$ of an element $R \in \tau_{h}$.

By virtue of the definition of the $V_{h}^{p}$ - and $V_{h}^{a}$-approximations, we have

$$
\begin{aligned}
I_{1} \equiv & \sum_{R \in \tau_{h}} \int_{\partial R} a_{1} \frac{\partial u}{\partial x} v n_{1} d S \\
= & \sum_{R \in \tau_{h} \text { face } F \subset \partial R} \sum_{F} a_{1} \frac{\partial u}{\partial x}\left[v-T_{F}(v)\right] n_{1} d S \\
= & \sum_{R \in \tau_{h} \text { face } F \subset \partial R} \sum_{F}\left(a_{1}-f_{R} a_{1} d x d y d z\right) \frac{\partial u}{\partial x}\left[v-T_{F}(v)\right] n_{1} d S \\
& \quad+\sum_{R \in \tau_{h} \text { face } F \subset \partial R} \sum_{R}\left(f_{R} a_{1} d x d y d z\right) \int_{F} \frac{\partial u}{\partial x}\left[v-T_{F}(v)\right] n_{1} d S \\
\equiv & I_{4}+I_{5} .
\end{aligned}
$$

It follows from the Cauchy-Schwarz inequality, Lemma 3.4 and Lemma 3.5 that

$$
\begin{aligned}
\left|I_{4}\right| & \equiv\left|\sum_{R \in \tau_{h}} \sum_{\text {face } F \subset \partial R} \int_{F}\left(a_{1}-f_{R} a_{1} d x d y d z\right) \frac{\partial u}{\partial x}\left[v-T_{F}(v)\right] n_{1} d S\right| \\
& \leq C h\|u\|_{2, \Omega}\|v\|_{1, h} .
\end{aligned}
$$

To estimate $I_{5}$, we first consider the $V_{h}^{a}$-approximation. In this case, since $T_{F}(v)=$ $f_{F} v d S$, by the Cauchy-Schwarz inequality and Lemma 3.4, we have

$$
\begin{aligned}
\left|I_{5}\right| & \equiv\left|\sum_{R \in \tau_{h}} \sum_{\text {face } F \subset \partial R}\left(f_{R} a_{1} d x d y d z\right) \int_{F} \frac{\partial u}{\partial x}\left[v-T_{F}(v)\right] n_{1} d S\right| \\
& =\left|\sum_{R \in \tau_{h}} \sum_{F \subset \partial R}\left(f_{R} a_{1} d x d y d z\right) \int_{F}\left[\frac{\partial u}{\partial x}-T_{F}\left(\frac{\partial u}{\partial x}\right)\right]\left[v-T_{F}(v)\right] n_{1} d S\right| \\
& \leq C h\|u\|_{2, \Omega}\|v\|_{1, h} .
\end{aligned}
$$


In the case of the $V_{h}^{p}$-approximation, we fix an element $R=[a-r, a+r] \times$ $[b-s, b+s] \times[c-t, c+t] \in \tau_{h}$ and consider its two opposite faces $F_{ \pm}=\{a \pm r\}$ $\times[b-s, b+s] \times[c-t, c+t]$ with $n_{1}=n_{ \pm}= \pm 1$. We then have by $(2.10)$, by the Cauchy-Schwarz inequality, and by the quasi-uniformity of the partitions $\tau_{h}$ that

$$
\begin{aligned}
\mid \int_{F_{+}} & \frac{\partial u}{\partial x}\left[v-T_{F_{+}}(v)\right] n_{+} d S+\int_{F_{-}} \frac{\partial u}{\partial x}\left[v-T_{F_{-}}(v)\right] n_{-} d S \mid \\
= & \mid \int_{b-s}^{b+s} \int_{c-t}^{c+t} \frac{\partial u(a+r, y, z)}{\partial x}[v(a+r, y, z)-v(a+r, b, c)] d y d z \\
& \quad-\int_{b-s}^{b+s} \int_{c-t}^{c+t} \frac{\partial u(a-r, y, z)}{\partial x}[v(a-r, y, z)-v(a-r, b, c)] d y d z \mid \\
= & \mid \int_{b-s}^{b+s} \int_{c-t}^{c+t}\left[\frac{\partial u(a+r, y, z)}{\partial x}-\frac{\partial u(a-r, y, z)}{\partial x}\right] \\
& \cdot\left[\int_{b}^{y} \frac{\partial v\left(a+r, y^{\prime}, z\right)}{\partial y} d y^{\prime}+\int_{c}^{z} \frac{\partial v\left(a+r, b, z^{\prime}\right)}{\partial z} d z^{\prime}\right] d y d z \mid \\
\leq & C h^{-2}\|u\|_{2,1, R}\|v\|_{1,1, R} \leq C h\|u\|_{2, R}\|v\|_{1, R} .
\end{aligned}
$$

Consequently, by rearranging the terms in the summation $I_{5}$, we obtain the estimate for the $V_{h}^{p}$-approximation

$$
\left|I_{5}\right| \leq C h\|u\|_{2, \Omega}\|v\|_{1, h} .
$$

It follows from (3.14)-(3.18) that

$$
\left|I_{1}\right| \leq C h\|u\|_{2, \Omega}\|v\|_{1, h} .
$$

Similar estimates hold for $I_{2}$ and $I_{3}$. We then obtain by (3.11) and (3.13) the following estimate

$$
\begin{aligned}
\left|d_{h}\left(u, v_{h}\right)\right| & \equiv\left|a_{h}\left(u, v_{h}\right)-\left(\mathcal{L} u, v_{h}\right)\right| \\
& \leq C h\|u\|_{2, \Omega}\left\|v_{h}\right\|_{1, h}, \quad \forall v_{h} \in V_{0 h} .
\end{aligned}
$$

This, together with (3.10) and (3.12), leads to (3.3) with $m=1$. The first part of the proof of the theorem is finished.

Proof of the $L^{2}$ error estimate. We follow the nonconforming version of the AubinNitsche argument [21,23]. Let $g \in L^{2}(\Omega)$. By Theorem 1.1, there exists a unique $\varphi_{g} \in H_{0}^{1}(\Omega) \cap H^{2}(\Omega)$ such that

$$
\begin{array}{rlrl}
L \varphi_{g} & =g, & & \text { in } \Omega, \\
u=0, & & \text { on } \partial \Omega,
\end{array}
$$

which by Theorem 1.1 satisfies

$$
\left\|\varphi_{g}\right\|_{2, \Omega} \leq C\|g\|_{0, \Omega}
$$


It is easy to verify, for any $\psi_{h} \in V_{0 h}$, that

$$
\left(u-u_{h}, g\right)=a_{h}\left(u-u_{h}, \varphi_{g}-\psi_{h}\right)-d_{h}\left(u, \varphi_{g}-\psi_{h}\right)-d_{h}\left(\varphi_{g}, u-u_{h}\right) .
$$

Consequently,

$$
\begin{aligned}
\left\|u-u_{h}\right\|_{0, \Omega}= & \sup _{0 \neq g \in L^{2}(\Omega)} \frac{\left(u-u_{h}, g\right)}{\|g\|_{0, \Omega}} \\
\leq \sup _{0 \neq g \in L^{2}(\Omega)} \frac{1}{\|g\|_{0, \Omega}} \inf _{h} \in V_{0 h} & {\left[\left|a_{h}\left(u-u_{h}, \varphi_{g}-\psi_{h}\right)\right|\right.} \\
& \left.\quad+\left|d_{h}\left(u, \varphi_{g}-\psi_{h}\right)\right|+\left|d_{h}\left(\varphi_{g}, u-u_{h}\right)\right|\right] .
\end{aligned}
$$

Fix $g \in L^{2}(\Omega)$. Let $W_{0 h} \subset C(\bar{\Omega}) \cap H_{0}^{1}(\Omega)$ be the trilinear finite element space over the partition $\tau_{h}$. Denote by $Q_{h}: C(\bar{\Omega}) \longrightarrow W_{0 h}$ the corresponding trilinear interpolation operator. We choose $\psi_{h}=I_{h}\left(Q_{h} \varphi_{g}\right) \in V_{0 h}$. By the $H_{h}^{1}$ error estimate, Theorem 3.1, the well-known interpolation properties of the operator $Q_{h}$ [7], and (3.21), we have

$$
\begin{aligned}
\left|a_{h}\left(u-u_{h}, \varphi_{g}-\psi_{h}\right)\right| & \leq C\left\|u-u_{h}\right\|_{1, h}\left\|\varphi_{g}-\psi_{h}\right\|_{1, h} \\
& \leq C h\|u\|_{2, \Omega}\left(\left\|\varphi_{g}-Q_{h} \varphi_{g}\right\|_{1, \Omega}+\left\|Q_{h} \varphi_{g}-I_{h}\left(Q_{h} \varphi_{g}\right)\right\|_{1, h}\right) \\
(3.23) & \leq C h^{2}\|u\|_{2, \Omega}\left(\left\|\varphi_{g}\right\|_{2, \Omega}+\left\|Q_{h} \varphi_{g}\right\|_{2, h}\right) \leq C h^{2}\|u\|_{2, \Omega}\|g\|_{0, \Omega} .
\end{aligned}
$$

Since $Q_{h} \varphi_{g} \in H_{0}^{1}(\Omega)$ and $u \in H_{0}^{1}(\Omega) \cap H^{2}(\Omega)$ is the solution to (1.2), by denoting $\chi_{h}=Q_{h} \varphi_{g}-\psi_{h}$, we have

$$
\begin{aligned}
d_{h}\left(u, \varphi_{g}-\psi_{h}\right) & =d_{h}\left(u, Q_{h} \varphi_{g}-\psi_{h}\right) \\
& =\sum_{R \in \tau_{h}} \int_{\partial R}\left[a_{1} \frac{\partial u}{\partial x} \chi_{h} n_{1}+a_{2} \frac{\partial u}{\partial y} \chi_{h} n_{2}+a_{3} \frac{\partial u}{\partial z} \chi_{h} n_{3}\right] d S \\
& \equiv J_{1}+J_{2}+J_{3} .
\end{aligned}
$$

On a face $F \subset \partial R$ for an element $R \in \tau_{h}$, we have by (3.9) that $f_{F} \chi_{h} d S=0$. Hence, since $Q_{h} \varphi_{g} \in C(\bar{\Omega})$, by the same argument as in the proof of the $H_{h}^{1}$ error estimate (cf. (3.14), (3.16)) we have

$$
\begin{aligned}
J_{1} \equiv & \sum_{R \in \tau_{h}} \int_{\partial R} a_{1} \frac{\partial u}{\partial x} \chi_{h} n_{1} d S=\sum_{R \in \tau_{h} \text { face } F \subset \partial R} \int_{F} a_{1} \frac{\partial u}{\partial x} \chi_{h} n_{1} d S \\
= & \sum_{R \in \tau_{h} \text { face } F \subset \partial R} \int_{F}\left(a_{1}-\bar{a}_{1}^{R}\right) \frac{\partial u}{\partial x} \chi_{h} n_{1} d S \\
& +\sum_{R \in \tau_{h} \text { face } F \subset \partial R} \bar{a}_{1}^{R} \int_{F}\left(\frac{\partial u}{\partial x}-f_{F} \frac{\partial u}{\partial x} d S\right) \chi_{h} n_{1} d S \\
= & \sum_{R \in \tau_{h} \text { face } F \subset \partial R} \int_{F}\left(a_{1}-\bar{a}_{1}^{R}\right) \frac{\partial u}{\partial x}\left(\chi_{h}-f_{F} \chi_{h} d S\right) n_{1} d S \\
& +\sum_{R \in \tau_{h} \text { face } F \subset \partial R} \sum_{a_{1}^{R}} \int_{F}\left(\frac{\partial u}{\partial x}-f_{F} \frac{\partial u}{\partial x} d S\right)\left(\chi_{h}-f_{F} \chi_{h} d S\right) n_{1} d S
\end{aligned}
$$


where

$$
\bar{a}_{1}^{R}=f_{R} a_{1} d x d y d z .
$$

Therefore, by Lemma 3.4, the Cauchy-Schwarz inequality, Theorem 3.1, the properties of the operator $Q_{h}$, and (3.21), we get

$$
\begin{aligned}
& \left|J_{1}\right| \leq C \sum_{R \in \tau_{h}} \sum_{\text {face } F \in \partial R}\left(h\|\nabla u\|_{0, F}+\left\|\frac{\partial u}{\partial x}-f_{F} \frac{\partial u}{\partial x} d S\right\|_{0, F}\right)\left\|\chi_{h}-f_{F} \chi_{h} d S\right\|_{0, F} \\
& \quad \leq C h\|u\|_{2, \Omega}\left\|\chi_{h}\right\|_{1, h} \leq C h^{2}\|u\|_{2, \Omega}\|\| \varphi_{g}\left\|_{2, \Omega} \leq C h^{2}\right\| u\left\|_{2, \Omega}\right\| g \|_{0, \Omega} .
\end{aligned}
$$

Similarly,

$$
\left|J_{2}\right|+\left|J_{3}\right| \leq C h^{2}\|u\|_{2, \Omega}\|g\|_{0, \Omega} .
$$

Now it follows from the fact $Q_{h} u \in H_{0}^{1}(\Omega)$ that

$$
d_{h}\left(\varphi_{g}, u-u_{h}\right)=d_{h}\left(\varphi_{g}, Q_{h} u-I_{h} Q_{h} u\right)+d_{h}\left(\varphi_{g}, I_{h} Q_{h} u-u_{h}\right) \equiv J_{4}+J_{5} .
$$

By the same argument used in estimating $d_{h}\left(u, \varphi_{g}-\psi_{h}\right)$ (cf. (3.24)-(3.26)), we obtain

$$
\begin{aligned}
\left|J_{4}\right| & \equiv\left|d_{h}\left(\varphi_{g}, Q_{h} u-I_{h} Q_{h} u\right)\right| \\
& \leq C h^{2}\left\|\varphi_{g}\right\|_{2, \Omega}\left\|Q_{h} u\right\|_{2, h} \leq C h^{2}\|g\|_{0, \Omega}\|u\|_{2, \Omega} .
\end{aligned}
$$

Denote $v=I_{h} Q_{h} u-u_{h} \in V_{0 h}$. Replacing $u$ by $\varphi_{g}$ in (3.19), by the $H_{h}^{1}$ error estimate, Theorem 3.1, the known properties of the operator $Q_{h}$ and (3.21), we then have

$$
\begin{aligned}
\left|J_{5}\right| & \equiv\left|d_{h}\left(\varphi_{g}, v\right)\right| \leq C h\left\|\varphi_{g}\right\|_{2, \Omega}\|v\|_{1, h} \\
& \leq C h\|g\|_{0, \Omega}\left(\left\|I_{h} Q_{h} u-Q_{h} u\right\|_{1, h}+\left\|Q_{h} u-u\right\|_{1, \Omega}+\left\|u-u_{h}\right\|_{1, h}\right) \\
& \leq C h^{2}\|u\|_{2, \Omega}\|g\|_{0, \Omega} .
\end{aligned}
$$

Finally, the $L^{2}$ error estimate (3.3) with $m=0$ is a direct consequence of (3.22)(3.29).

\section{Connection with multilinear finite elements}

In the previous proof, we made use of the piecewise linear function $I_{h} Q_{h} u$ several times as an approximation function of $u$. This makes some connection between the considered nonconforming elements and the conforming multilinear elements. Furthermore, it is in fact true that all the piecewise linear functions in $V_{0 h}$ approximate the solution $u$ well enough.

To be more precise, let $W_{0 h} \subset C(\bar{\Omega}) \cap H_{0}^{1}(\Omega)$ be again the trilinear finite element space over the mesh $\tau_{h}$. By the proof of Lemma 3.6, we know that all the functions in the subspace $I_{h} W_{0 h} \subset V_{0 h}$ are piecewise linear functions. Furthermore, by taking the boundary condition into account, it is easy to see that the operator $I_{h}: W_{0 h} \longrightarrow I_{h} W_{0 h}$ is in fact one-to-one and onto. Thus, the subspace $I_{h} W_{0 h} \subset V_{0 h}$ 
has the same number of degrees of freedom as the trilinear finite element space $W_{0 h}$ does. Now by the Lax-Milgram lemma, there exists a unique $\bar{u}_{h} \in I_{h} W_{0 h}$ such that

$$
a_{h}\left(\bar{u}_{h}, v_{h}\right)=\left(f, v_{h}\right), \quad \forall v_{h} \in I_{h} W_{0 h} .
$$

We realize that $\bar{u}_{h}$ is in fact the projection of the finite element solution $u_{h} \in V_{0 h}$ into the subspace $I_{h} W_{0 h}$, since by (3.2) and (4.1), we have

$$
a_{h}\left(u_{h}-\bar{u}_{h}, v_{h}\right)=0, \quad \forall v_{h} \in I_{h} W_{0 h} .
$$

Now let us write the error

$$
u-\bar{u}_{h}=\left(u-I_{h} u_{h}^{t}\right)+\left(I_{h} u_{h}^{t}-\bar{u}_{h}\right),
$$

where $u_{h}^{t} \in W_{0 h}$ is the trilinear finite element approximation of $u$, i.e.,

$$
a\left(u_{h}^{t}, w_{h}\right)=\left(f, w_{h}\right), \quad \forall w_{h} \in W_{0 h} .
$$

By the known results on this approximation $u_{h}^{t}$ [7], and by Theorem 3.1, we get

$$
\left\|u-I_{h} u_{h}^{t}\right\|_{1, h} \leq\left\|u-u_{h}^{t}\right\|_{1, \Omega}+\left\|u_{h}^{t}-I_{h} u_{h}^{t}\right\|_{1, h} \leq C h\|u\|_{2, \Omega} .
$$

Notice that $\lambda_{h} \equiv I_{h} u_{h}^{t}-\bar{u}_{h} \in I_{h} W_{0 h} \subset V_{0 h}$. By (3.1), (4.5), (3.19) and the known results on $u_{h}^{t}$, we have

$$
\begin{aligned}
\alpha\left\|\lambda_{h}\right\|_{1, h}^{2} & \leq a_{h}\left(\lambda_{h}, \lambda_{h}\right)=a_{h}\left(I_{h} u_{h}^{t}-u, \lambda_{h}\right)+a_{h}\left(u-\bar{u}_{h}, \lambda_{h}\right) \\
& \leq C\left\|I_{h} u_{h}^{t}-u\right\|_{1, h}\left\|\lambda_{h}\right\|_{1, h}+\left|d_{h}\left(u, \lambda_{h}\right)\right| \leq C h\|u\|_{2, \Omega}\left\|\lambda_{h}\right\|_{1, h} .
\end{aligned}
$$

It follows from (4.3)-(4.6) that

$$
\left\|u-\bar{u}_{h}\right\|_{1, h} \leq C h\|u\|_{2, \Omega} .
$$

Now, by the definition of $d_{h}(\cdot, \cdot)$ (cf. (3.11)) and (4.2), we have for any $g \in L^{2}(\Omega)$ and any $\psi_{h} \in I_{h} W_{0 h}$ that

$$
\begin{aligned}
\left(u-\bar{u}_{h}, g\right) & =\left(u-u_{h}, g\right)+\left(u_{h}-\bar{u}_{h}, g\right) \\
& =\left(u-u_{h}, g\right)+a_{h}\left(u_{h}-\bar{u}_{h}, \varphi_{g}-\psi_{h}\right)-d_{h}\left(\varphi_{g}, u_{h}-\bar{u}_{h}\right) .
\end{aligned}
$$

Setting $\psi_{h}=I_{h} Q_{h} \varphi_{g} \in I_{h} W_{0 h}$ in (4.8), we have

$$
\begin{array}{r}
\quad\left|a_{h}\left(u_{h}-\bar{u}_{h}, \varphi_{g}-\psi_{h}\right)\right| \leq C\left\|u_{h}-\bar{u}_{h}\right\|_{1, h}\left\|\varphi_{g}-I_{h} Q_{h} \varphi_{g}\right\|_{1, h} \\
\leq C\left(\left\|u_{h}-u\right\|_{1, h}+\left\|u-\bar{u}_{h}\right\|_{1, h}\right)\left(\left\|\varphi_{g}-Q_{h} \varphi_{g}\right\|_{1, h}+\left\|Q_{h} \varphi_{g}-I_{h} Q_{h} \varphi_{g}\right\|_{1, h}\right) \\
\leq C h^{2}\|u\|_{2, \Omega}\left(\left\|\varphi_{g}\right\|_{2, \Omega}+\left\|Q_{h} \varphi_{g}\right\|_{2, \Omega}\right) \leq C h^{2}\|u\|_{2, \Omega}\|g\|_{0, \Omega},
\end{array}
$$

where we used the known properties of $Q_{h}$, Theorem 3.1, Theorem 3.3, (4.7) and (3.21). Replacing $u$ by $\varphi_{g}$ in (3.19), we then get by (3.21), Theorem 3.1 and (4.7) that

$$
\begin{aligned}
\left|d_{h}\left(\varphi_{g}, u_{h}-\bar{u}_{h}\right)\right| & \leq C h\left\|\varphi_{g}\right\|_{2, \Omega}\left\|u_{h}-\bar{u}_{h}\right\|_{1, h} \\
& \leq C h\|g\|_{0, \Omega}\left(\left\|u_{h}-u\right\|_{1, h}+\left\|u-\bar{u}_{h}\right\|_{1, h}\right) \\
& \leq C h^{2}\|u\|_{2, \Omega}\|g\|_{0, \Omega} .
\end{aligned}
$$

We have in fact proved, by (4.7)-(4.10) and Theorem 3.3, the following

Theorem 4.1. Let $u \in H_{0}^{1}(\Omega) \cap H^{2}(\Omega)$ and $\bar{u}_{h} \in I_{h} W_{0 h}$ be the solutions to (1.2) and (4.1), respectively. Then,

$$
\left\|u-\bar{u}_{h}\right\|_{0, \Omega}+h\left\|u-\bar{u}_{h}\right\|_{1, h} \leq C h^{2}\|u\|_{2, \Omega} .
$$




\section{A SUPERCONVERGENCE ESTIMATE}

We first give a superconvergence estimate for the interpolation error gradients. Denote by $C_{R}$ the center of a rectangular element $R \in \tau_{h}$.

Lemma 5.1. For any $R \in \tau_{h}$, we have

$$
\left|\nabla\left(v-I_{h} v\right)\left(C_{R}\right)\right| \leq C h^{2}|v|_{3, \infty, R}, \quad \forall v \in W^{3, \infty}(R) .
$$

Proof. Let $\hat{R}=[-1,1] \times[-1,1] \times[-1,1]$. Define: $\hat{F}: W^{3, \infty}(\hat{R}) \longrightarrow \mathbb{R}$ by

$$
\hat{F}(\hat{v})=\partial_{\xi}(\hat{v}-\hat{I} \hat{v})(O), \quad \hat{v} \in W^{3, \infty}(\hat{R})
$$

where $O=(0,0,0)$ and $\hat{I}$ is the interpolation operator over $\hat{R}$ for the considered elements. By the imbedding $W^{3, \infty}(\hat{R}) \hookrightarrow C^{1}(\hat{R})$, we have

$$
|\hat{F}(\hat{v})| \leq C\|\hat{v}\|_{3, \infty, \hat{R}}, \quad \hat{v} \in W^{3, \infty}(\hat{R})
$$

Now the basis functions for our elements over $\hat{R}$ can be easily obtained by setting $a=b=c=0$ and $r=s=t=1$ in (2.4) and (2.6), respectively. By their properties (cf. (2.3), (2.7)) and by the Taylor expansion, a series of calculations then lead to

$$
\hat{F}(\hat{p})=0, \quad \forall \hat{p} \in P_{2}(\hat{R})
$$

where $P_{2}(\hat{R})$ is the set of all polynomials over $\hat{R}$ with degrees at most 2 . It follows from (5.2), (5.3) and the Bramble-Hilbert lemma that

$$
|\hat{F}(\hat{v})| \leq C|\hat{v}|_{3, \infty, \hat{R}}, \quad \hat{v} \in W^{3, \infty}(\hat{R})
$$

This, together with the affine transformation from $\hat{R}$ to $R$ (cf. (3.7)), leads to

$$
\left|\partial_{x}\left(v-I_{h} v\right)\left(C_{R}\right)\right| \leq C h^{-1}|\hat{F}(\hat{v})| \leq C h^{2}|v|_{3, \infty, R}
$$

Similar estimates hold for $\partial_{y}\left(v-I_{h} v\right)$ and $\partial_{z}\left(v-I_{h} v\right)$.

In the rest of this section, we will only consider the $V_{h}^{a}$-approximation, i.e., the averaged element approximation, to the solution of the model problem

$$
\begin{aligned}
-\Delta u & =f, & & \text { in } \Omega, \\
u & =0, & & \text { on } \partial \Omega .
\end{aligned}
$$

The following result shows that the nonconforming error functional $d_{h}(\cdot, \cdot)$, as defined in (3.11), is of one order higher than usual. Hence the nonconformity, in this case, is weak. 
Lemma 5.2. Let $u \in H_{0}^{1}(\Omega) \cap W^{3, \infty}(\Omega)$ be the solution to (5.4). Then,

$$
\left|d_{h}\left(u, v_{h}\right)\right| \leq C h^{2}\|u\|_{3, \infty, \Omega}\left\|v_{h}\right\|_{1, h}, \quad \forall v_{h} \in V_{0 h}^{a} .
$$

Proof. Fix $v=v_{h} \in V_{0 h}^{a}$. We have as before that

$$
d_{h}(u, v)=\sum_{R \in \tau_{h}} \int_{\partial R}\left(\frac{\partial u}{\partial x} v n_{1}+\frac{\partial u}{\partial y} v n_{2}+\frac{\partial u}{\partial z} v n_{3}\right) d S \equiv K_{1}+K_{2}+K_{3} .
$$

By virtue of the $V_{0 h}^{a}$-approximation, we can further write

$$
\begin{aligned}
K_{1} & \equiv \sum_{R \in \tau_{h}} \int_{\partial R} \frac{\partial u}{\partial x} v n_{1} d S \\
& =\sum_{R \in \tau_{h} \text { face } F \in \partial R} \int_{F}\left[\frac{\partial u}{\partial x}-\frac{\partial u\left(M_{F}\right)}{\partial x}\right]\left[v-T_{F}(v)\right] n_{1} d S,
\end{aligned}
$$

where $M_{F}$ is the center of a face $F$. Fix $R=[a-r, a+r] \times[b-s, b+s] \times[c-t, c+t] \in \tau_{h}$ and consider its two opposite faces $F_{ \pm}=\{a \pm r\} \times[b-s, b+s] \times[c-t, c+t]$ with $n_{1}=n_{ \pm}= \pm 1$. An application of the Bramble-Hilbert lemma leads to the estimate

$$
\int_{R}\left[\frac{\partial^{2} u(x, y, z)}{\partial x^{2}}-\frac{\partial^{2} u(x, b, c)}{\partial x^{2}}\right]^{2} d x d y d z \leq C h^{5}\|u\|_{3, \infty, R}^{2} .
$$

It then follows from (2.10), the Cauchy-Schwarz inequality, (5.8) and Lemma 3.5, that

$$
\begin{aligned}
\mid \int_{F_{+}} & {\left[\frac{\partial u}{\partial x}-\frac{\partial u\left(M_{F_{+}}\right)}{\partial x}\right]\left[v-T_{F_{+}}(v)\right] n_{+} d S } \\
& +\int_{F_{-}}\left[\frac{\partial u}{\partial x}-\frac{\partial u\left(M_{F_{-}}\right)}{\partial x}\right]\left[v-T_{F_{-}}(v)\right] n_{-} d S \mid \\
= & \mid \int_{b-s}^{b+s} \int_{c-t}^{c+t}\left\{\left[\frac{\partial u(a+r, y, z)}{\partial x}-\frac{\partial u(a+r, b, c)}{\partial x}\right]\right. \\
& \left.\quad-\left[\frac{\partial u(a-r, y, z)}{\partial x}-\frac{\partial u(a-r, b, c)}{\partial x}\right]\right\}\left[v(a+r, y, z)-T_{F_{+}}(v)\right] d y d z \mid \\
= & \mid \int_{b-s}^{b+s} \int_{c-t}^{c+t}\left\{\int_{a-r}^{a+r}\left[\frac{\partial^{2} u(x, y, z)}{\partial x^{2}}-\frac{\partial^{2} u(x, b, c)}{\partial x^{2}}\right] d x\right\} \\
\leq & C h^{\frac{7}{2}}\|u\|_{3, \infty, \Omega}\|v\|_{1, R} .
\end{aligned}
$$

Consequently, by a rearrangement of the terms in the summation $K_{1}$, we have

$$
\left|K_{1}\right| \leq C h^{\frac{7}{2}}\|u\|_{3, \infty, \Omega} \sum_{R \in \tau_{h}}\|v\|_{1, R} \leq C h^{2}\|u\|_{3, \infty, \Omega}\|v\|_{1, h},
$$

where we also used the Cauchy-Schwarz inequality and the fact that

$$
\left|\tau_{h}\right| \equiv \sum_{R \in \tau_{h}} 1 \leq C h^{-3}
$$

In the two-dimensional case, $h^{-3}$ should be replaced by $h^{-2}$. Similar estimates hold for $K_{2}$ and $K_{3}$ as well. Hence, (5.5) follows. 
Lemma 5.3. Let $u \in H_{0}^{1}(\Omega) \cap W^{3, \infty}(\Omega)$ be the solution to (5.4) and $u_{h} \in V_{0 h}^{a}$ its $V_{0 h}^{a}$-approximation. Assume that all the elements in $\tau_{h}$ are cubes. Then

$$
\left\|I_{h} u-u_{h}\right\|_{1, h} \leq C h^{2}\|u\|_{3, \infty, \Omega} .
$$

Proof. Denote $\gamma_{h} \equiv I_{h} u-u_{h} \in V_{0 h}^{a}$. Since each element in $\tau_{h}$ is assumed to be a cube, it is easy to see that $\gamma_{h}$ is piecewise harmonic. On the other hand, since $I_{h}=I_{h}^{a}$ is the interpolation operator for the $V_{h}^{a}$-approximation, we have

$$
\int_{F}\left(I_{h} u-u\right) d S=0, \quad \forall \text { faces } F \subset \partial R, \forall R \in \tau_{h} .
$$

It then follows from (2.10) that

$$
\begin{aligned}
a_{h}\left(I_{h} u-u, \gamma_{h}\right)= & \sum_{R \in \tau_{h}} \int_{R} \nabla\left(I_{h} u-u\right) \nabla \gamma_{h} d x d y d z \\
= & \sum_{R \in \tau_{h} \text { face } F \in \partial R}\left[\int_{F}\left(I_{h} u-u\right) \frac{\partial \gamma_{h}}{\partial x} n_{1} d S\right. \\
& \left.+\int_{F}\left(I_{h} u-u\right) \frac{\partial \gamma_{h}}{\partial y} n_{2} d S+\int_{F}\left(I_{h} u-u\right) \frac{\partial \gamma_{h}}{\partial z} n_{3} d S\right]=0 .
\end{aligned}
$$

Now, by (3.1), (5.11) and Lemma 5.2, we have

$$
\begin{aligned}
\alpha\left\|\gamma_{h}\right\|_{1, h}^{2} & \leq a_{h}\left(\gamma_{h}, \gamma_{h}\right)=a_{h}\left(I_{h} u-u_{h}, \gamma_{h}\right) \\
& =a_{h}\left(u-u_{h}, \gamma_{h}\right)=d_{h}\left(u, \gamma_{h}\right) \leq C h^{2}\|u\|_{3, \infty, \Omega}\left\|\gamma_{h}\right\|_{1, h},
\end{aligned}
$$

leading to (5.10).

Now we present the main result in this section.

Theorem 5.4. With the same assumption as in Lemma 5.3, we have

$$
\left[\sum_{R \in \tau_{h}}\left|\nabla\left(u-u_{h}\right)\left(C_{R}\right)\right|^{2} h^{3}\right]^{\frac{1}{2}} \leq C h^{2}\|u\|_{3, \infty, \Omega} .
$$

Proof. By Lemma 5.1, Theorem 3.2 and Lemma 5.3, we have

$$
\begin{aligned}
& {\left[\sum_{R \in \tau_{h}} \mid \nabla\right.}\left.\left.\left(u-u_{h}\right)\left(C_{R}\right)\right|^{2} h^{3}\right]^{\frac{1}{2}} \\
& \leq C\left[\sum_{R \in \tau_{h}}\left|\nabla\left(u-I_{h} u\right)\left(C_{R}\right)\right|^{2} h^{3}+\sum_{R \in \tau_{h}}\left|\nabla\left(I_{h} u-u_{h}\right)\left(C_{R}\right)\right|^{2} h^{3}\right]^{\frac{1}{2}} \\
& \leq C\left[h^{4}\|u\|_{3, \infty, \Omega}^{2}+h^{3} \sum_{R \in \tau_{h}}\left(h^{-\frac{3}{2}}\left\|I_{h} u-u_{h}\right\|_{1, R}\right)^{2}\right]^{\frac{1}{2}} \\
& \leq C h^{2}\|u\|_{3, \infty, \Omega},
\end{aligned}
$$

completing the proof. 
Now let us turn back to both the $V_{h}^{p}$ - and $V_{h}^{a}$-approximations with general partitions of the solution to the general problem (1.1). Recall that $Q_{h}: C(\bar{\Omega}) \longrightarrow$ $W_{0 h}$ is the interpolation operator for the trilinear finite element. We can easily obtain, if the solution $u$ is smooth, that

$$
\left|\nabla\left(u-Q_{h} u\right)\left(C_{R}\right)\right|+\left|\nabla\left(u-I_{h} Q_{h} u\right)\left(C_{R}\right)\right|=O\left(h^{2}\right) .
$$

As discussed in $\S 4$, the considered elements are connected with the conforming multilinear finite elements through the subspace $I_{h} W_{0 h}$. Thus, the estimate (5.13), Lemma 5.1 and the known superconvergence results (cf. [20]) on the multilinear elements naturally lead to a conjecture on the pointwise superconvergence estimates for the error of the gradient: if the solution $u$ is smooth enough and the partitions $\tau_{h}$ are suitably regular, then

$$
\max _{R \in \tau_{h}}\left|\nabla\left(u-u_{h}\right)\left(C_{R}\right)\right|=O\left(h^{2}\right)
$$

In its discrete average form, the superconvergence estimate (5.12) for the simplest case makes the estimate (5.14) believable somewhat. However, compared with a recent work on higher-order error estimates on the nonconforming Wilson finite element [4], the proof or disproof of (5.14) will be more difficult since our elements do not have any conforming counterparts, though there is some connection between our elements and the conforming multilinear elements.

\section{EFFECT OF NUMERICAL INTEGRATION}

We define on the reference element $\hat{R}=[-1,1] \times[-1,1] \times[-1,1]$ the numerical integration scheme

$$
\int_{\hat{R}} \hat{g}(\xi, \eta, \zeta) d \xi d \eta d \zeta \doteq \sum_{i=1}^{I} \hat{\omega}_{i} \hat{g}\left(\hat{Q}_{i}\right), \quad \hat{g} \in C(\hat{R})
$$

where $\hat{\omega}_{i}>0, \hat{Q}_{i} \equiv\left(\xi_{i}, \eta_{i}, \zeta_{i}\right) \in \hat{R}, i=1, \ldots, I$, and $I$ is a positive integer. Let us denote

$$
\hat{P}=\operatorname{Span}\left\{1, \xi, \eta, \zeta, \xi^{2}-\eta^{2}, \xi^{2}-\zeta^{2}\right\} .
$$

We shall assume that the quadrature scheme is exact on $\hat{P}$, i.e.,

$$
\int_{\hat{R}} \hat{p}(\xi, \eta, \zeta) d \xi d \eta d \zeta=\sum_{i=1}^{I} \hat{\omega}_{i} \hat{p}\left(\hat{Q}_{i}\right), \quad \hat{p} \in \hat{P},
$$

and that the set of quadrature points

$$
\left\{\hat{Q}_{i}\right\}_{i=1}^{I} \text { in }(6.1) \text { contains a } P_{1}(\hat{R}) \text {-unisolvent subset, }
$$

where $P_{1}(\hat{R})$ is the set of all linear polynomials over $\hat{R}$. 
The conditions (6.2) and (6.3) are satisfied by the quadrature schemes

$\begin{cases}\text { Scheme 1: } & I=6, \text { all } \hat{w}_{i}=\frac{4}{3},\left\{\hat{Q}_{i}\right\}_{i=1}^{6}=\{( \pm \hat{q}, 0,0),(0, \pm \hat{q}, 0),(0,0, \pm \hat{q})\} \\ \text { Scheme 2: } & I=8, \text { all } \hat{w}_{i}=1,\left\{\hat{Q}_{i}\right\}_{i=1}^{8}=\{(\xi, \eta, \zeta): \xi, \eta, \zeta= \pm \hat{q}\},\end{cases}$

where $0<\hat{q} \leq 1$. The computations for the dynamics of martensitic microstructure reported in [18] used Scheme 1 with $\hat{q}=1$, in which case the nodes of the quadrature scheme are identical to the nodes of the finite element with respect to the $V_{h}^{p}$ approximation. Scheme 2 with $\hat{q}=1 / \sqrt{3}$ is the Gaussian quadrature over $\hat{R}$ with eight nodes of quadrature.

Now, for an element $R \equiv[a-r, a+r] \times[b-s, b+s] \times[c-t, c+t] \in \tau_{h}$, let $K_{R}: \hat{R} \longrightarrow R$ be the invertible affine mapping given by (3.7). Then, the quadrature scheme (6.1) induces automatically the following quadrature scheme over the element $R \in \tau_{h}$,

$$
\int_{R} g(x, y, z) d x d y d z \doteq \sum_{i=1}^{I} \omega_{i, R} g\left(Q_{i, R}\right), \quad g \in C(R),
$$

where

$$
\omega_{i, R}=\operatorname{det}\left(\nabla K_{R}\right) \hat{w}_{i}, \quad Q_{i, R}=K_{R}\left(\hat{Q}_{i}\right), \quad i=1, \ldots, I .
$$

To apply the numerical quadrature to the finite element formulation (3.2), in what follows we assume that in (1.1) $c \in C(\bar{\Omega})$ and $f \in C(\bar{\Omega})$. Let us now define $a_{h}^{*}(\cdot, \cdot): V_{h} \times V_{h} \longrightarrow \mathbb{R}$ by

$$
\begin{aligned}
a_{h}^{*}\left(v_{h}, w_{h}\right)= & \sum_{R \in \tau_{h}} \sum_{i=1}^{I} \omega_{i, R}\left[\left(a_{1} \frac{\partial v_{h}}{\partial x} \frac{\partial w_{h}}{\partial x}\right)\left(Q_{i, R}\right)+\left(a_{2} \frac{\partial v_{h}}{\partial y} \frac{\partial w_{h}}{\partial y}\right)\left(Q_{i, R}\right)\right. \\
& \left.+\left(a_{3} \frac{\partial v_{h}}{\partial z} \frac{\partial w_{h}}{\partial z}\right)\left(Q_{i, R}\right)+\left(c v_{h} w_{h}\right)\left(Q_{i, R}\right)\right], \quad v_{h}, w_{h} \in V_{h}
\end{aligned}
$$

and define $f_{h}^{*}: V_{h} \longrightarrow \mathbb{R}$ by

$$
f_{h}^{*}\left(v_{h}\right)=\sum_{R \in \tau_{h}} \sum_{i=1}^{I} \omega_{i, R}\left(f v_{h}\right)\left(Q_{i, R}\right), \quad v_{h} \in V_{h} .
$$

Obviously, $a_{h}^{*}(\cdot, \cdot)$ and $f_{h}^{*}(\cdot)$ are discrete approximations for $a_{h}(\cdot, \cdot)$ and $f_{h}(\cdot) \equiv$ $(f, \cdot)$, respectively.

By the uniform $V_{0 h}$-ellipticity of $a_{h}(\cdot, \cdot)$ given by $(3.1)$ and the conditions $(6.2)$ and (6.3), we have the following uniform $V_{0 h^{-}}$-ellipticity of $a_{h}^{*}(\cdot, \cdot)$ (cf. Theorem 4.1 .2 in $[7])$.

Lemma 6.1. There exists a constant $\alpha^{*}>0$, independent of $h$, such that

$$
a_{h}^{*}\left(v_{h}, v_{h}\right) \geq \alpha^{*}\left\|v_{h}\right\|_{1, h}^{2}, \quad \forall v_{h} \in V_{0 h} .
$$

It is now a direct consequence of the Lax-Milgram lemma that there exists a unique $u_{h}^{*} \in V_{0 h}$, the discrete solution to (1.2), such that

$$
a_{h}^{*}\left(u_{h}^{*}, v_{h}\right)=f_{h}^{*}\left(v_{h}\right), \quad \forall v_{h} \in V_{0 h} .
$$

Our main result in this section is that, with a certain smoothness of the coefficients in (1.1), the discrete solution $u_{h}^{*} \in V_{0 h}$ converges to the exact solution $u \in H_{0}^{1}(\Omega) \cap H^{2}(\Omega)$ with the same rates as the solution $u_{h} \in V_{0 h}$ does. 
Theorem 6.2. Assume that in (1.1), in addition, $c \in W^{1, \infty}(\Omega)$ and $f \in W^{1, \infty}(\Omega)$. Let $u \in H_{0}^{1}(\Omega) \cap H^{2}(\Omega)$ and $u_{h}^{*} \in V_{0 h}$ be the solutions to (1.2) and (6.9), respectively. Then,

$$
\left\|u-u_{h}^{*}\right\|_{1, h} \leq C h\|f\|_{1, \infty, \Omega} .
$$

Theorem 6.3. If the coefficients $a_{1}, a_{2}, a_{3}$, and $c$, and the term $f$ are all in $W^{2, \infty}(\Omega)$, then

$$
\left\|u-u_{h}^{*}\right\|_{0, \Omega} \leq C h^{2}\|f\|_{2, \infty, \Omega} .
$$

To prove these two theorems, we need to estimate the errors induced by the quadrature schemes (6.1) and (6.4). Thus, we first define the quadrature error functionals

$$
\hat{E}(\hat{g}) \equiv \int_{\hat{R}} \hat{g} d \xi d \eta d \zeta-\sum_{i=1}^{I} \hat{\omega}_{i} \hat{g}\left(\hat{Q}_{i}\right), \quad \hat{g} \in C(\hat{R})
$$

and

$$
E_{R}(g) \equiv \int_{R} g d x d y d z-\sum_{i=1}^{I} \omega_{i, R} g\left(Q_{i, R}\right), \quad g \in C(R), \quad R \in \tau_{h}
$$

Obviously,

$$
E_{R}(g)=\operatorname{det}\left(\nabla K_{R}\right) \hat{E}(\hat{g}), \quad \hat{g}=g \circ K_{R} \in C(\hat{R}) .
$$

Recall that $P_{R}$ is the finite element polynomial space over the element $R \in \tau_{h}$ (cf. (2.1)).

Lemma 6.4. Let $a_{1}, a_{2}, a_{3}$ and $c$ be given in (1.1). Suppose $c \in W^{1, \infty}(\Omega)$. Then, for any $R \in \tau_{h}$ and any $v, w \in P_{R}$, we have

$$
\begin{gathered}
\left|E_{R}\left(a_{1} \frac{\partial v}{\partial x} \frac{\partial w}{\partial x}\right)\right|+\left|E_{R}\left(a_{2} \frac{\partial v}{\partial y} \frac{\partial w}{\partial y}\right)\right|+\left|E_{R}\left(a_{3} \frac{\partial v}{\partial z} \frac{\partial w}{\partial z}\right)\right|+\left|E_{R}(c v w)\right| \\
\leq C h\|v\|_{2, R}\|w\|_{1, R} .
\end{gathered}
$$

Proof. Let $R \equiv[a-r, a+r] \times[b-s, b+s] \times[c-t, c+t]$. As before, let the mapping $K_{R}: \hat{R} \longrightarrow R$ be defined by (3.7). Write $\hat{\varphi}=\varphi \circ K_{R}$ for $\varphi \in W^{1, \infty}(R)$. Since the $L^{\infty}$ and $L^{2}$ norms are equivalent on the finite-dimensional space $P_{\hat{R}}$, we have that

$$
|\hat{E}(\hat{\varphi} \hat{p})| \leq C\|\hat{\varphi}\|_{1, \infty, \hat{R}}\|\hat{p}\|_{0, \hat{R}}, \quad \forall \hat{\varphi} \in W^{1, \infty}(\hat{R}), \hat{p} \in P_{\hat{R}} .
$$

Replacing $\hat{\varphi}$ in (6.16) by $\hat{\varphi}+\hat{c}$ with $\hat{c}$ an arbitrary constant, by (6.2) we obtain

$$
\begin{aligned}
|\hat{E}(\hat{\varphi} \hat{p})| & \leq C \inf _{\hat{c}=\text { constant }}\|\hat{\varphi}+\hat{c}\|_{1, \infty, \hat{R}}\|\hat{p}\|_{0, \hat{R}} \\
& \leq C|\hat{\varphi}|_{1, \infty, \hat{R}}\|\hat{p}\|_{0, \hat{R}}, \quad \forall \hat{\varphi} \in W^{1, \infty}(\hat{R}), \hat{p} \in P_{\hat{R}} .
\end{aligned}
$$

Now let $a \in\left\{a_{1}, a_{2}, a_{3}, c\right\}, q \in\left\{v, \frac{\partial v}{\partial x}, \frac{\partial v}{\partial y}\right\}$, and $p \in\left\{w, \frac{\partial w}{\partial x}, \frac{\partial w}{\partial y}\right\}$, where $v, w \in P_{R}$. Note that $\frac{\partial w}{\partial x}, \frac{\partial w}{\partial y} \in P_{R}$ if $w \in P_{R}$. Setting $\hat{\varphi}=\hat{a} \hat{q}$ in (6.17), by (6.14), (3.7) and Theorem 3.2 , we get

$$
\begin{aligned}
\left|E_{R}(a q p)\right| & \leq C h^{3}|\hat{E}(\hat{a} \hat{q} \hat{p})| \leq C h^{3}|\hat{a} \hat{q}|_{1, \infty, \hat{R}}\|\hat{p}\|_{0, \hat{R}} \leq C h^{\frac{5}{2}}\|a q\|_{1, \infty, R}\|p\|_{0, R} \\
& \leq C h^{\frac{5}{2}}\|q\|_{1, \infty, R}\|p\|_{0, R} \leq C h\|q\|_{1, R}\|p\|_{0, R} \leq C h\|v\|_{2, R}\|w\|_{1, R} .
\end{aligned}
$$

This proves (6.15). 
Lemma 6.5. Suppose $f \in W^{1, \infty}(\Omega)$. Then, for any $R \in \tau_{h}$ and any $v \in P_{R}$, we have

$$
\left|E_{R}(f v)\right| \leq C h^{\frac{5}{2}}\|f\|_{1, \infty, R}\|v\|_{1, R}
$$

Proof. Since $\hat{E}(\hat{\varphi})=0$ for any constant polynomial $\hat{\varphi}$, by the Bramble-Hilbert lemma we have

$$
|\hat{E}(\hat{\varphi})| \leq C|\hat{\varphi}|_{1, \infty, \hat{R}}, \quad \forall \hat{\varphi} \in W^{1, \infty}(\hat{R}) .
$$

Taking $\hat{\varphi}=\hat{f} \hat{v}$, by (6.14) and Theorem 3.2 we have

$$
\begin{aligned}
\left|E_{R}(f v)\right| & \leq C h^{3}|\hat{E}(\hat{f} \hat{v})| \leq C h^{3}|\hat{f} \hat{v}|_{1, \infty \hat{R}} \leq C h^{4}|f v|_{1, \infty, R} \\
& \leq C h^{4}\|f\|_{1, \infty, R}\|v\|_{1, \infty, R} \leq C h^{\frac{5}{2}}\|f\|_{1, \infty, R}\|v\|_{1, R},
\end{aligned}
$$

completing the proof.

Lemma 6.6. Let $R \in \tau_{h}$ and $a \in W^{2, \infty}(R)$. Then for any $v, w \in P_{R}$ we have

$$
\begin{aligned}
& \left|E_{R}(a v)\right| \leq C h^{\frac{7}{2}}\|a\|_{2, \infty, R}\|v\|_{2, R}, \\
& \left|E_{R}(a v w)\right| \leq C h^{2}\|a\|_{2, \infty, R}\|v\|_{2, R}\|w\|_{2, R} .
\end{aligned}
$$

Proof. By $(6.12)$ and the imbedding $H^{2}(\hat{R}) \hookrightarrow C(\hat{R})$, we have

$$
|\hat{E}(\hat{a} \hat{v})| \leq C\|\hat{a} \hat{v}\|_{2, \hat{R}} .
$$

By (6.14), (6.2), the Bramble-Hilbert lemma, and (3.7), we thus get

$$
\left|E_{R}(a v)\right| \leq C h^{3}|\hat{a} \hat{v}|_{2, \hat{R}} \leq C h^{\frac{7}{2}}|a v|_{2, R} \leq C h^{\frac{7}{2}}\|a\|_{2, \infty, R}\|v\|_{2, R},
$$

obtaining (6.20). Now, replacing $a$ in (6.20) by aw, we have, by Theorem 3.2, that

$$
\begin{aligned}
\left|E_{R}(a v w)\right| & \leq C h^{\frac{7}{2}}\|a w\|_{2, \infty, R}\|v\|_{2, R} \leq C h^{\frac{7}{2}}\|a\|_{2, \infty, R}\|w\|_{2, \infty, R}\|v\|_{2, R} \\
& \leq C h^{2}\|a\|_{2, \infty, R}\|v\|_{2, R}\|w\|_{2, R},
\end{aligned}
$$

leading to (6.21).

Notice that in the two-dimensional case, the orders $h^{\frac{5}{2}}$ in (6.19) and $h^{\frac{7}{2}}$ in (6.20) should be replaced by $h^{2}$ and $h^{3}$, respectively.

Proof of Theorem 6.2. By (6.8), (3.2), (6.9), Lemma 6.4, and Lemma 6.5, we have

$$
\begin{aligned}
\alpha^{*} \| u_{h}- & u_{h}^{*} \|_{1, h}^{2} \leq a_{h}^{*}\left(u_{h}-u_{h}^{*}, u_{h}-u_{h}^{*}\right) \\
& =\left[a_{h}^{*}\left(u_{h}, u_{h}-u_{h}^{*}\right)-a_{h}\left(u_{h}, u_{h}-u_{h}^{*}\right)\right]+\left[f_{h}\left(u_{h}-u_{h}^{*}\right)-f_{h}^{*}\left(u_{h}-u_{h}^{*}\right)\right] \\
& \leq C h \sum_{R \in \tau_{h}}\left\|u_{h}\right\|_{2, R}\left\|u_{h}-u_{h}^{*}\right\|_{1, R}+C h^{\frac{5}{2}} \sum_{R \in \tau_{h}}\|f\|_{1, \infty, R}\left\|u_{h}-u_{h}^{*}\right\|_{1, R} \\
& \leq C h\left(\left\|u_{h}\right\|_{2, h}+\|f\|_{1, \infty, \Omega}\right)\left\|u_{h}-u_{h}^{*}\right\|_{1, h},
\end{aligned}
$$


where we also used the Cauchy-Schwarz inequality and (5.9). It then follows from Theorem 3.1-Theorem 3.3 and Theorem 1.1 that

$$
\begin{aligned}
\left\|u-u_{h}^{*}\right\|_{1, h} & \leq\left\|u-u_{h}\right\|_{1, h}+\left\|u_{h}-u_{h}^{*}\right\|_{1, h} \\
& \leq C h\|u\|_{2, \Omega}+C h\left(\left\|u_{h}-I_{h} u\right\|_{2, h}+\left\|I_{h} u-u\right\|_{2, h}+\|f\|_{1, \infty, \Omega}\right) \\
& \leq C h\left(\|u\|_{2, \Omega}+\|f\|_{1, \infty, \Omega}\right) \leq C h\|f\|_{1, \infty, \Omega} .
\end{aligned}
$$

which is the result of Theorem 6.2.

Proof of Theorem 6.3. For any $g \in L^{2}(\Omega)$, by (3.2) and (6.9), the following identity holds,

$$
\begin{gathered}
\left(u_{h}-u_{h}^{*}, g\right)=a_{h}\left(u_{h}-u_{h}^{*}, \varphi_{g_{h}}-\psi_{h}\right)-\left[a_{h}\left(u_{h}^{*}, \psi_{h}\right)-a_{h}^{*}\left(u_{h}^{*}, \psi_{h}\right)\right] \\
+\left[f_{h}\left(\psi_{h}\right)-f_{h}^{*}\left(\psi_{h}\right)\right], \quad \forall \psi_{h} \in V_{0 h},
\end{gathered}
$$

where $\varphi_{g_{h}} \in V_{0 h}$ satisfies

$$
a_{h}\left(\varphi_{g_{h}}, v_{h}\right)=\left(g, v_{h}\right), \quad \forall v_{h} \in V_{0 h},
$$

and $\varphi_{g} \in H_{0}^{1}(\Omega) \cap H^{2}(\Omega)$ satisfies (3.20) and (3.21). Consequently,

$$
\begin{aligned}
\left\|u-u_{h}^{*}\right\|_{0, \Omega} \leq & \left\|u-u_{h}\right\|_{0, h}+\sup _{0 \neq g \in L^{2}(\Omega)} \frac{1}{\|g\|_{0, \Omega}} \inf _{\psi_{h} \in V_{0 h}}\left[\left|a_{h}\left(u_{h}-u_{h}^{*}, \varphi_{g_{h}}-\psi_{h}\right)\right|\right. \\
& \left.+\left|a_{h}\left(u_{h}^{*}, \psi_{h}\right)-a_{h}^{*}\left(u_{h}^{*}, \psi_{h}\right)\right|+\left|f_{h}\left(\psi_{h}\right)-f_{h}^{*}\left(\psi_{h}\right)\right|\right] .
\end{aligned}
$$

Now let us fix $g \in L^{2}(\Omega)$ and choose $\psi_{h}=I_{h} \varphi_{g}$. By Theorem 3.1, Theorem 6.2, Theorem 3.3 and (3.21), we get

$$
\begin{aligned}
\left|a_{h}\left(u_{h}-u_{h}^{*}, \varphi_{g_{h}}-\psi_{h}\right)\right| & \leq C\left\|u_{h}-u_{h}^{*}\right\|_{1, h}\left\|\varphi_{g_{h}}-\psi_{h}\right\|_{1, h} \\
& \leq C h^{2}\|f\|_{1, \infty, \Omega}\|g\|_{0, \Omega} .
\end{aligned}
$$

It follows from the definitions of $a_{h}(\cdot, \cdot), a_{h}^{*}(\cdot, \cdot)$, and $E_{R}(\cdot)$ that

$$
\begin{aligned}
\left|a_{h}\left(u_{h}^{*}, \psi_{h}\right)-a_{h}^{*}\left(u_{h}^{*}, \psi_{h}\right)\right| \leq \sum_{R \in \tau_{h}} & {\left[\left|E_{R}\left(a_{1} \frac{\partial u_{h}^{*}}{\partial x} \frac{\partial \psi_{h}}{\partial x}\right)\right|+\left|E_{R}\left(a_{2} \frac{\partial u_{h}^{*}}{\partial y} \frac{\partial \psi_{h}}{\partial y}\right)\right|\right.} \\
+ & \left.\left|E_{R}\left(a_{3} \frac{\partial u_{h}^{*}}{\partial z} \frac{\partial \psi_{h}}{\partial z}\right)\right|+\left|E_{R}\left(c u_{h}^{*} \psi_{h}\right)\right|\right] .
\end{aligned}
$$

Notice that $\left.\partial u_{h}^{*}\right|_{R},\left.\partial \psi_{h}\right|_{R} \in P_{R}$, for $R \in \tau_{h}$, where $\partial=\partial_{x}, \partial_{y}$ or $\partial_{z}$. Therefore, by Lemma 6.6, the Cauchy-Schwarz inequality, Theorem 6.2, (3.21) and (1.3), we have

$$
\begin{aligned}
\left|a_{h}\left(u_{h}^{*}, \psi_{h}\right)-a_{h}^{*}\left(u_{h}^{*}, \psi_{h}\right)\right| & \leq C h^{2} \sum_{R \in \tau_{h}}\left\|u_{h}^{*}\right\|_{2, R}\left\|\psi_{h}\right\|_{2, R} \leq C h^{2}\left\|u_{h}^{*}\right\|_{2, h}\left\|I_{h} \varphi_{g}\right\|_{2, h} \\
& \leq C h^{2}\left(\|u\|_{2, \Omega}+\|f\|_{1, \infty, \Omega}\right)\|g\|_{0, \Omega} \\
& \leq C h^{2}\|f\|_{1, \infty, \Omega}\|g\|_{0, \Omega} .
\end{aligned}
$$

By Lemma 6.6, (5.9), Theorem 3.1 and (3.21), we have

$$
\begin{aligned}
\left|f_{h}\left(\psi_{h}\right)-f_{h}^{*}\left(\psi_{h}\right)\right| & \leq C \sum_{R \in \tau_{h}}\left|E_{R}\left(f \psi_{h}\right)\right| \leq C h^{\frac{7}{2}} \sum_{R \in \tau_{h}}\|f\|_{2, \infty, R}\left\|\psi_{h}\right\|_{2, R} \\
& \leq C h^{2}\|f\|_{2, \infty, \Omega}\left\|\psi_{h}\right\|_{2, h} \leq C h^{2}\|f\|_{2, \infty, \Omega}\|g\|_{0, \Omega} .
\end{aligned}
$$

Now (6.11) is a direct consequence of the combination of (6.24)-(6.28) and Theorem 3.3. The proof is complete. 


\section{REFERENCES}

1. R. A. Adams, Sobolev Spaces, Academic Press, New York, 1975. MR 56:9247

2. J. M. Ball and R. D. James, Fine phase mixtures as minimizers of energy, Arch. Rational Mech. Anal. 100 (1987), 13-52. MR 89c:80005

3. J. M. Ball and R. D. James, Proposed experimental tests of a theory of fine microstructure and the two-well problem, Phil. Trans. R. Soc. Lond. A 338 (1992), 389-450.

4. H. S. Chen and B. Li, Superconvergence analysis and error expansion for the Wilson nonconforming finite element, Numer. Math. 69 (1994), 125-140. MR 95k:65105

5. C. Chipot, C. Collins, and D. Kinderlehrer, Numerical analysis of oscillations in multiple well problems, Numer. Math. 70 (1995), 259-282. CMP 95:12

6. C. Chipot and C. Collins, Numerical approximation in variational problems with potential wells, SIAM J. Numer. Anal. 29 (1992), 1002-1019. MR 93j:65171

7. P. G. Ciarlet, The Finite Element Method for Elliptic Problems, North-Holland, Amsterdam, 1978. MR 58:25001

8. C. Collins, Computation of twinning, in Microstructure and Phase Transitions (James, Kinderlehrer, and Luskin, eds.), IMA Volumes in Mathematics and its Applications, vol. 54, SpringerVerlag, New York, 1993, pp. 39-50. CMP 95:09

9. C. Collins and M. Luskin, The computation of the austenitic-martensitic phase transition, Partial Differential Equations and Continuum Models of Phase Transitions, Lecture Notes in Physics (M. Rascle, D.Serre, and M.Slemrod, eds.), vol. 344, Springer-Verlag, 1989, pp. 34-50. MR 90k:80007

10. C. Collins and M. Luskin, Optimal order error estimates for the finite element approximation of the solution of a nonconvex variational problem, Math. Comp. 57 (1991), 621-637. MR 92a:65276

11. C. Collins, D. Kinderlehrer, and M. Luskin, Numerical approximation of the solution of a variational problem with a double well potential, SIAM J. Numer. Anal. 28 (1991), 321-332. MR 92c:73015

12. C. Collins, M. Luskin, and J. Riordan, Computational results for a two-dimensional model of crystalline microstructure, in Microstructure and Phase Transitions (James, Kinderlehrer, and Luskin, eds.), IMA Volumes in Mathematics and its Applications, vol. 54, Springer-Verlag, New York, 1993, pp. 51-56. CMP 95:09

13. J. L. Ericksen, Some constrained elastic crystals, Material Instabilities in Continuum Mechanics and Related Problems, (J. M. Ball, ed.), Oxford Univ. Press, 1987, pp. 119-137. MR 90a: 73145

14. J. L. Ericksen, Constitutive theory for some constrained elastic crystals, Int. J. Solids and Structures 22 (1986), 951-964.

15. D. Gilbarg and N. S. Trudinger, Elliptic Partial Differential Equations of Second Order, 2nd ed., Springer-Verlag, Berlin and New York, 1983. MR 86c:35035

16. P. Grisvard, Elliptic Problems in Nonsmooth Domains, Monographs and Studies in Mathematics 24, Pitman, Boston, London, Melbourne, 1985. MR 86m:35044

17. D. Kinderlehrer, Remarks about equilibrium configurations of crystals, Material Instabilities in Continuum Mechanics and Related Problems, J. M. Ball, ed., Oxford University Press, 1987, pp. 217-242. CMP 21:04

18. P. Klouček and M. Luskin, The computation of the dynamics of martensitic microstructure, Continuum Mech. Thermodyn. 6 (1994), 209-240. MR 95d:73009

19. R. Kohn, Relaxation of a double-well energy, Continuum Mech. and Thermodyn. 3 (1991), 193-236. MR 93d:73014

20. M. Kř́̌žek and P. Neittaanmäki, On superconvergence techniques, Acta Appl. Math. 9 (1987), 175-198. MR 88h:65208

21. P. Lascaux and P. Lesaint, Some nonconforming finite elements for the plate bending problem, Rev. Française Automat. Informat. Recherche Opérationelle Sér. Rouge Anal. Numér. 9 (1975), 9-53. MR 54:11941

22. M. Luskin and L. Ma, Analysis of the finite element approximation of microstructure in micromagnetics, SIAM J. Numer. Anal. 29 (1992), 320-331. MR 92m:65147

23. J. A. Nitsche, Convergence of nonconforming methods, Proc. Symp. on the Mathematical Aspects of Finite Elements in Partial Differential Equations (C. de Boor, ed.), Academic Press, New York, 1974, pp. 15-53. MR 58:31923 
24. R. Rannacher and S. Turek, Simple nonconforming quadrilateral Stokes element, Numer. Meth. for PDEs 8 (1992), 97-111. MR 92i:65170

25. G. Strang and G. J. Fix, An Analysis of the Finite Element Method, Prentice-Hall, Englewood Cliffs, New Jersey, 1973. MR 56:1747

206 Church St. SE, School of Mathematics, University of Minnesota, Minneapolis, MinNESOTA 55455

E-mail address: kloucek@math.umn.edu

E-mail address: bli@math.umn.edu

E-mail address: luskin@math.umn.edu 